\title{
Saturation Transfer Difference NMR spectroscopy using glycopolymers
}

Janet Muzulu and Amit Basu*

Department of Chemistry, Brown University, Providence RI, USA 02912

abasu@brown.edu

\begin{abstract}
We report the use of Saturation Transfer Difference (STD) NMR spectroscopy to observe the interaction of various phenylboronic acids (PBAs) with synthetic glycopolymers presenting galactose and glucose. After optimizing experimental parameters to maximize spin diffusion, the binding of boronic acids to the glycopolymers was examined using STD NMR. Efficient amplification factor build-up curves which were used to generate an epitope map for the boronic acid binding to the glycopolymers. STD-NMR was also used to detect the interaction between indole and a galactosylated glycopolymer, providing an indole-based view of this $\mathrm{C}-\mathrm{H}-\pi$ interaction.
\end{abstract}




\section{Introduction}

Ligand-based NMR experiments such as saturation transfer difference (STD) spectroscopy have been widely used for detecting protein-ligand binding interactions. ${ }^{1-5}$ These experiments are useful for characterizing medium-to-low affinity $(\mu \mathrm{M}-\mathrm{mM})$ interactions and can provide additional information about the mode of ligand binding. ${ }^{1} \mathrm{H}$ STD NMR relies on the spin diffusion from saturated protons in the macromolecule followed by transfer of the saturation to the bound ligand. STD-NMR has also been applied to study the binding of small molecules to nonproteinogenic macromolecules such as chromatographic supports ${ }^{6,7}$, inorganic nanoparticles ${ }^{8-10}$, colloidal dispersions, ${ }^{11}$ as well as nucleic acids ${ }^{12,13}$. A recent report describes the use of STD-NMR to confirm an interaction between a polyacrylamide glycopolymer and a 42 -mer peptide, ${ }^{14}$ but to the best of our knowledge the use of STD NMR to generate epitope maps for polymer-bound ligands has not been reported to date. The slow molecular tumbling rate of glycopolymers leads to short $T_{2}$ relaxation times and broad peaks in the solution NMR, making them suitable candidates for the STD method. Additionally, glycopolymers also have high proton density, especially along the polymer backbone, which should allow spin diffusion through a dipolar-coupled network of spins in the macromolecules..$^{15,16}$

In this manuscript we report the use of STD-NMR to observe the interaction of various substituted phenylboronic acids (PBAs) with synthetic glycopolymers of galactose (Gal90) and glucose (Glc90) (Figure 1) in solution. PBAs bind monosaccharides with $\mathrm{mM}$ or high $\mu \mathrm{M}$ affinity via the formation of boronate esters. ${ }^{17-19}$ We also report the use of STD-NMR to detect the interaction of indole with a glycopolymer via a non-covalent $\mathrm{C}-\mathrm{H}-\pi$ interaction. ${ }^{20-25}$ The work described here thus represents a first step toward examining small molecule binding to glycopolymers using STD-NMR. 


\section{Materials and Methods}

All reagents and solvents were purchased from commercial suppliers and used as received. Dry solvents (methanol and dichloromethane) were obtained using a commercially available solvent purification system from GlassContour based on reported purification protocols. ${ }^{26}$ Reactions were carried out under nitrogen atmosphere. Centrifugation of precipitated polymers was carried out using an Adams physician's compact centrifuge. Regenerated cellulose dialysis membrane 1000 MWCO (6 Spectra/Por) from Spectrum was used for dialysis. TLC Silica Gel 60 F254 plates from EMD Chemicals were used. ${ }^{1} \mathrm{H}$ NMR experiments of the polymer backbone and glycopolymers were recorded on a Bruker Avance Ultra-Shield $400 \mathrm{MHz}$ with an automatic tuning and matching BBO probe. All STD and DOSY NMR spectra were recorded on a Bruker Avance Ultra-Shield $600 \mathrm{MHz}$ instrument at $296 \mathrm{~K}$ with an automatic tuning and matching BBO probe. Samples for STD and DOSY NMR were prepared by combining an appropriate amount of the PBAs (by volume) and glycopolymers. The final concentration of the PBAs in each NMR tube was $1 \mathrm{mM}$, while that of the glycopolymers was $100 \mu \mathrm{M}$ in a buffer of $0.1 \mathrm{M}$ sodium phosphate in deuterium oxide corrected to $\mathrm{pH}$ 7.2. STD and DOSY NMR experiments were carried out in duplicate. For indole studies, the final concentration of indole in the NMR tube was $3 \mathrm{mM}$, while that of Gal90 was $50 \mu \mathrm{M}$ in deuterium oxide with no buffer.

STD NMR ${ }^{27}$ STD experiments were performed using the stddifesgp.3 sequence from the Bruker pulse sequence library. Eight dummy scans were collected at the beginning of each STD experiment. The on-resonance irradiation of the glycopolymer was applied at $1.07 \mathrm{ppm}$. Offresonance irradiation was applied at $40 \mathrm{ppm}$. 1D STD NMR spectra were multiplied by an exponential line- broadening function of $2.5 \mathrm{~Hz}$ prior to Fourier transformation. The irradiation 
power of the selective pulses in the STD NMR experiment was set to $\left.(\gamma / 2 \pi) \mathrm{B}_{1}\right) 100 \mathrm{~Hz}$. Selective pre- saturation of the glycopolymer was achieved by a train of Gauss-shaped pulses of 25 ms length each, separated by a $1.5 \mathrm{~ms}$ delay. The saturation time was varied from $1 \mathrm{~s}$ to $8 \mathrm{~s}$. For both $1 \mathrm{D}{ }^{1} \mathrm{H}$ and STD experiments, the excitation sculpting with gradients water suppression sequence was employed. Spectra were processed using Bruker Topspin version 3.6.2 Software.

DOSY NMR ${ }^{28}$ The standard Bruker protocol was used with a $600 \mathrm{MHz}$ Avance spectrometer equipped with a broad-band z-gradient probe; 16 1D 1H spectra were collected with a gradient duration of 1-ms and an echo delay of $150 \mathrm{~ms}$ for the PBAs in the presence or absence of the glycopolymers. Acquisition times of 25 minutes were required for the oligosaccharide samples and complexes. The ledbpg2s pulse sequence, with stimulated echo, longitudinal eddy current compensation, bipolar gradient pulses, and two spoil gradients, was run with a linear gradient $\left(52.5 \mathrm{G}^{\mathrm{cm}}{ }^{-1}\right)$ stepped between $2 \%$ and $95 \%$. The $1 \mathrm{D}{ }^{1} \mathrm{H}$ spectra were processed and automatically baseline corrected. The diffusion dimension, zero-filled to $1 \mathrm{k}$, was exponentially fitted according to pre-set windows for the diffusion dimension. Comparison of diffusion coefficients in the presence or absence of glycopolymer was measured by taking the water signal as a standard. 


\section{Results and Discussion}
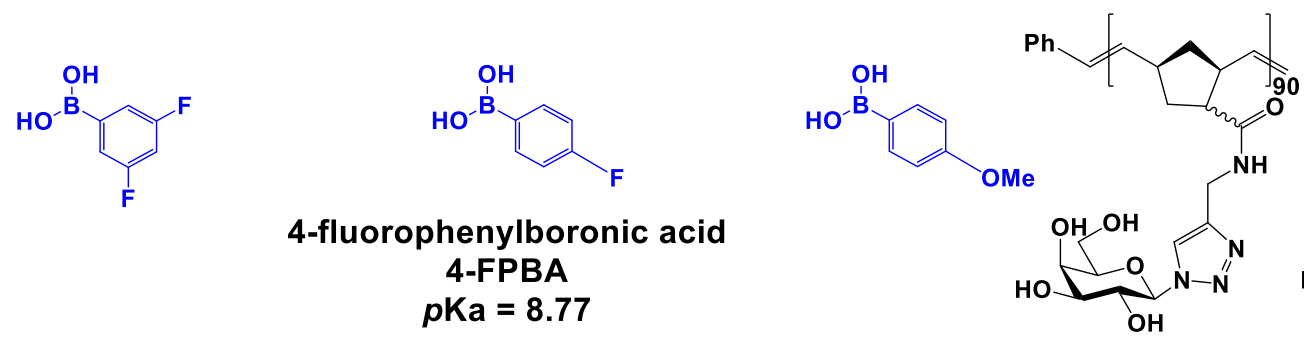
3,5-difluorophenylboronic acid 3,5-DFPBA pKa $=7.08$
4-methoxyphenylboronic acid 4-MPBA $p K a=9.3$

Gal90

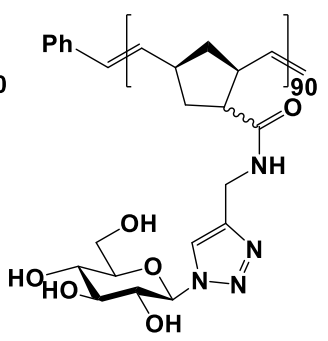

GIc90

Figure 1 - Structures of phenyl boronic acids and glycopolymers used in these studies

The STD experiment involves the acquisition of two separate spectra - one spectrum is obtained in an on-resonance experiment and the second in an off-resonance experiment. The offresonance experiment is a reference experiment in which saturation is performed at a frequency far from both the protein and ligand resonances. In the on-resonance experiment, resonances of the macromolecular receptor, in our case the glycopolymer, are selectively irradiated by applying an $r f$ pulse train for a given saturation time at a frequency where only receptor signals resonate. ${ }^{29}$ The resulting saturation spreads through the entire macromolecule by spin diffusion ${ }^{30}$ and is transferred to protons of the bound form of the ligand that are in close proximity to the macromolecular receptor. A difference STD spectrum, generated by subtraction of the onresonance spectrum from the off-resonance spectrum, exhibits signals for protons on the ligand that received saturation transfer from the protein. The degree of saturation of the individual ligand protons reflects their proximities to the receptor and can be used to obtain an epitope map ${ }^{27}$ that describes the ligand interactions. Our first objective was to determine the ability of the glycopolymers to be effectively saturated and undergo spin diffusion. 


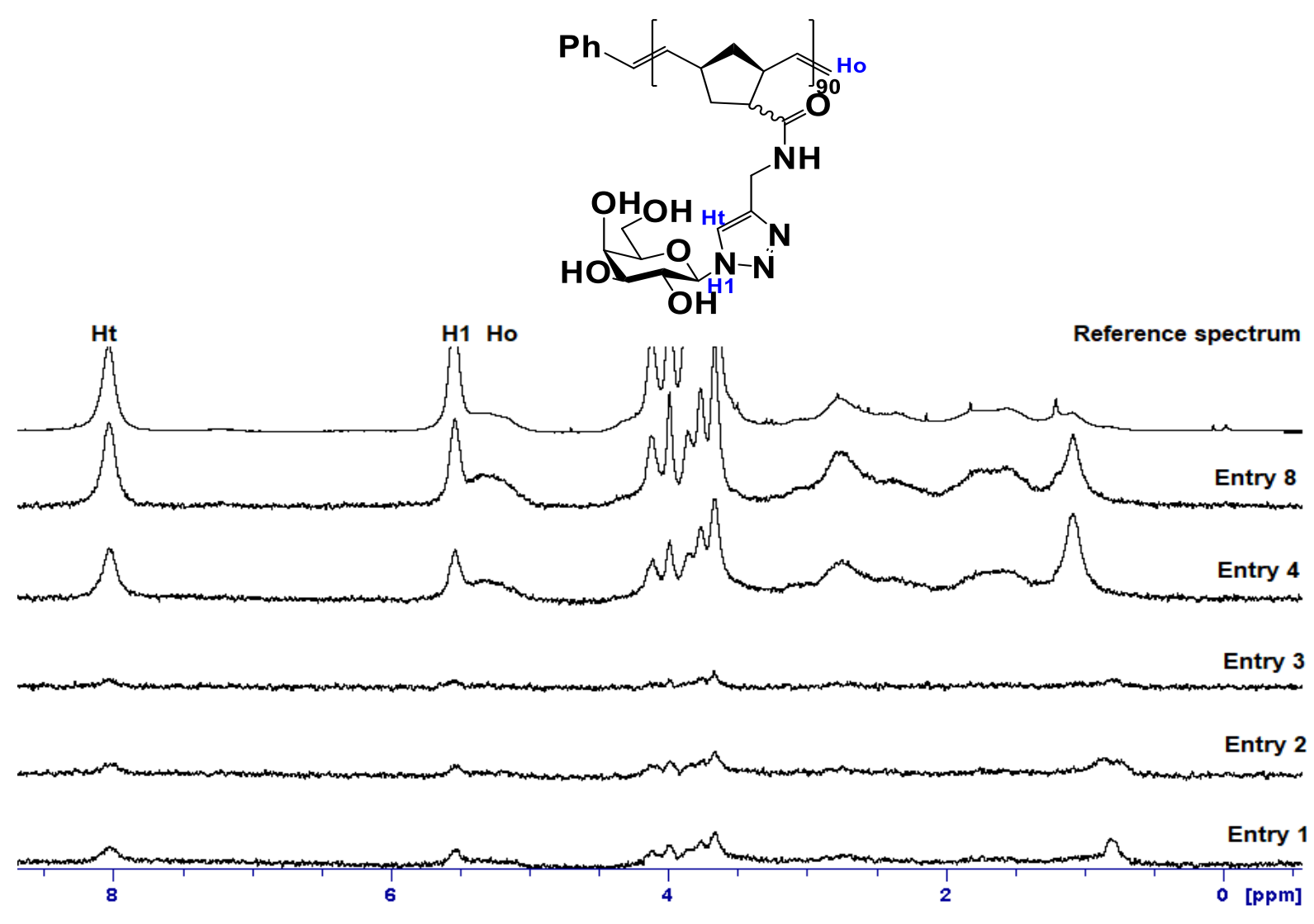

Figure 2 - The envelopes of the Gal90 STD spectra are similar to that of the reference spectrum indicating that the glycopolymer is effectively saturated. Experimental details for each entry are found in Table 1. All spectra are shown at the same vertical scale, with the exception of the reference spectrum, shown at $0.1 \mathrm{x}$ scale.

Optimal parameters for maximal saturation of Gal90 were obtained after optimizing the on-resonance frequency, pulse shape, pulse length, as well as pulse power, all at a fixed saturation time of $1 \mathrm{~s}$. The glycopolymers exhibit numerous resonances between $5 \mathrm{ppm}$ and $0.8 \mathrm{ppm}$ (Reference spectrum, Figure 2), a region outside of the spectral window of the PBAs, providing a wide window for the choice of the on-resonance saturation frequency. The intensities of the triazole proton of Gal90 at 8.05 ppm were monitored in each experiment. Efficient spin diffusion within the macromolecule leads to the saturation of the entire macromolecule and not just the irradiated resonances. ${ }^{27}$ The efficiency of spin diffusion was determined by first conducting the off-resonance experiment at a frequency distant from the Gal90 envelope ( $c a .40 \mathrm{ppm}$ ) to obtain 
the reference spectrum (Figure 2) of the glycopolymer, with an intensity $\left(I_{0}\right)$ at $8.05 \mathrm{ppm}$. The reference spectrum exhibits the broad resonances of the glycopolymer, along with smaller sharp peaks corresponding to low molecular weight impurities. The on-resonance experiment was then conducted using on-resonance frequencies located in the aliphatic region of Gal90, and triazole proton intensities $\left(I_{\mathrm{SAT}}\right)$ in the corresponding spectra were obtained. If the difference spectrum (off-resonance - on-resonance) exhibited an envelope similar to that of the reference spectrum, then $I_{\mathrm{SAT}}<I_{0}$, which indicates that effective intrapolymer saturation transfer was occurring via a dipolar-coupled network of spins in the glycopolymer. ${ }^{27}$ If the difference spectrum generated a flat line, then $I_{\text {SAT }}$ is equal to $I_{0}$, indicating that spin diffusion did not occur within the glycopolymer, and no STD effect was observed. The STD effect is calculated using equation $1 .^{10}$

$$
\mathrm{STD} \text { effect }=\frac{I_{0}-I_{S A T}}{I_{0}}(1)
$$

The default parameters from Bruker ${ }^{31}$ (Gaus shaped pulse of $50 \mathrm{~ms}$ length and pulse power of $-50 \mathrm{~dB}$ ) resulted in an STD effect of 0.034 upon irradiation at $0.8 \mathrm{ppm}$ (Table 1 Entry 1, Figure 2). Changing the pulse shape to EBurp or Reburp decreases the STD effect (Table 1 Entries 2 and 3, Figure 2). Changing the on-resonance frequency to $1.07 \mathrm{ppm}$ results in a 3 -fold increase in the STD effect, with a Gaus shaped pulse as the most effective (Table 1 Entries 4-6, Figure 2). Changing the pulse length from $50 \mathrm{~ms}$ to $25 \mathrm{~ms}$ results in a higher STD effect at either on resonance frequency (Table 1 Entries 7,8), with an STD effect almost 1.5 times greater than a 50 ms pulse at the same on-resonance (Entry 4 vs 8). This observation is consistent with prior reports that pulses shorter than $50 \mathrm{~ms}$ can result in stronger STD effects. ${ }^{32}$ The use of a $10 \mathrm{~ms}$ Gaus pulse marginally reduces the STD effect, and the use of a low power pulse $-70 \mathrm{~dB}$ results in no detectable spin diffusion (Table 1, Entries 9, 10). When a T1p spin lock filter of $50 \mathrm{~ms}$ was applied to the parameters of Entry 8 (25 ms long Gaus shaped pulse), the STD effect dropped from 0.13 to 0.10 
(Entry 12). A spin lock filter suppresses the broad resonances of the macromolecule in order to allow the small molecule resonances to be more clearly observed and to quantitate their intensities without interference from any overlapping macromolecule resonances. The parameters in entry 11 were adopted as the optimal parameters used for all subsequent experiments.

\begin{tabular}{llllll} 
Entry & $\begin{array}{l}\text { Shaped } \\
\text { pulse }\end{array}$ & $\begin{array}{l}\text { Pulse power/ } \\
\text {-dB }\end{array}$ & $\begin{array}{l}\text { Pulse length/ } \\
\text { ms }\end{array}$ & $\begin{array}{l}\text { On- } \\
\text { resonance/ppm }\end{array}$ & $\begin{array}{l}\text { STD } \\
\text { effect }\end{array}$ \\
\hline $\mathbf{1}$ & Gaus & 50 & 50 & 0.8 & 0.0341 \\
$\mathbf{2}$ & Eburp & 50 & 50 & 0.8 & 0.0218 \\
$\mathbf{3}$ & Reburp & 50 & 50 & 0.8 & 0.0114 \\
\hline $\mathbf{4}$ & Gaus & 50 & 50 & 1.07 & 0.0898 \\
$\mathbf{5}$ & Eburp & 50 & 50 & 1.07 & 0.0605 \\
$\mathbf{6}$ & Reburp & 50 & 50 & 1.07 & 0.0280 \\
\hline $\mathbf{7}$ & Gaus & 47.5 & 25 & 0.8 & 0.0582 \\
$\mathbf{8}$ & Gaus & 47.5 & 25 & 1.07 & 0.1297 \\
$\mathbf{9}$ & Gaus & 47.5 & 10 & 1.07 & 0.1294 \\
$\mathbf{1 0}$ & Gaus & 70 & 25 & 1.07 & 0 \\
$\mathbf{1 1}$ * & Gaus & $\mathbf{4 7 . 5}$ & $\mathbf{2 5}$ & $\mathbf{1 . 0 7}$ & $\mathbf{0 . 0 9 8 2}$
\end{tabular}

Table 1- Optimization of selective excitation pulses to maximize the STD effect of the glycopolymer. Each entry shows the parameters that were varied. The off-resonance irradiation was applied at $40 \mathrm{ppm}$. *Entry 11 - A T $1 p$ spin lock filter of $50 \mathrm{~ms}$ was applied

With optimized parameters in hand, the STD NMR experiment was carried out using 3,5difluoroboronic acid (3,5-DFPBA) in the presence of Gal90. A negative control experiment was performed to ensure that the excitation profile of the selected shaped pulse did not cause excitation of ligand resonances. Irradiation of 3,5-DFPBA using the optimized parameters did not elicit an STD effect, confirming that there was no excitation bleed-through to the ligand (Figure S6). Figure 4 shows stacked STD plots of the 3,5-DFPBA and the glycopolymer at saturation times varied from 1 to 8 seconds. The plots show an increase in the peak intensities of the aromatic resonances of 3,5-DFPBA with increasing saturation. This observation indicates that the PBA is undergoing saturation transfer from the Gal90, since the earlier control experiment had established that no 
ligand resonances are excited by the pulse sequence using in the experiment. The STD amplification factor (STD-AF) allows a better assessment of the absolute magnitude of the STD effect and can be quantified by multiplying equation (1) by the ligand to receptor ratio: ${ }^{33}$

$$
S T D-A F=\frac{I_{0}-I_{S A T}}{I_{0}} \times \frac{[L]}{[R]}
$$

where $[\mathrm{L}]$ is the ligand concentration, and $[\mathrm{R}]$ is the macromolecule concentration. Figure 5 (blue features) shows the STD effect for both the ortho and para protons of 3,5-DFPBA as a function of saturation time. Figure 4 also shows that Gal90 resonances receive insufficient background suppression. This phenomenon has also been previously observed with a small $\mathrm{RNA}^{34}$ and a membrane protein ${ }^{35}$. Using a relaxation filter to reduce or eliminate the RNA background signals was not possible because the T1p values required were too long. ${ }^{34}$ In our case, the signals of the 3,5-DFPBA can be quantified without problems since the Gal90 resonances do not overlap those of any of the PBAs.

Because the small molecule protons have different longitudinal relaxation rates $\left(T_{1}\right)$, this can produce artifacts in epitope mapping. Protons with slower $\mathrm{T}_{1}$ relaxations accumulate saturation in solution, giving rise to higher STD relative intensity, thereby overestimating their proximity to the macromolecule. ${ }^{36}$ Angulo et. al. proposed STD buildup curves at several saturation times to eliminate the $T_{1}$ bias at long saturation times. ${ }^{33}$ The build-up curves are fitted to equation 3 , a monoexponential function. ${ }^{33}$

$$
S T D-A F_{t s a t}=S T D-A F_{\max }\left(1-e^{-k_{s a t} \times t_{s a t}}\right)
$$

The term STD-AF $\max _{\max }$ corresponds to the plateau of each curve, $k_{s a t}$ is a rate constant that measures the speed of STD build-up, and $\mathrm{t}_{\text {sat }}$ is the saturation time. Values for $k_{s a t}$ and STD-AF max $_{\text {ax }}$ are derived by least-squares fitting, and their product yields the initial slope of the build-up curve at zero saturation time $\left(\mathrm{STD}-\mathrm{AF}_{0}\right)$. $\mathrm{STD}-\mathrm{AF}_{0}$ corresponds to the $\mathrm{STD}$ intensity without any $\mathrm{T}_{1}$ bias. The 
ligand protons that are positioned closest to the saturated receptor protons will exhibit the largest STD-AF as they will experience a greater degree of saturation transfer from the macromolecule. The STD-AF for these protons is set to $100 \%$, and the STD-AF values for all other ligand protons are scaled relative to it. The resulting values provide an epitope map of the small molecule, where lower percentages correspond to decreased proximity of those ligands protons from the receptor. ${ }^{27}$

Equation 3 was used to generate Figure 5, which displays STD-AF plotted against the saturation time for the ortho and para protons of 3,5-DFPBA (blue curves). The 3,5-DFPBA STD effect builds up with increasing saturation time, eventually reaching a plateau. The para proton exhibits a larger STD-AF at all saturation times compared to the ortho proton, with STD-AF values of 0.88 and 0.69 , respectively (Table $\mathrm{S} 11$ ). The higher $\mathrm{STD}_{-} \mathrm{AF}_{0}$ value observed for the para proton suggests that this proton lies in closer proximity to the polymer backbone upon complexation.
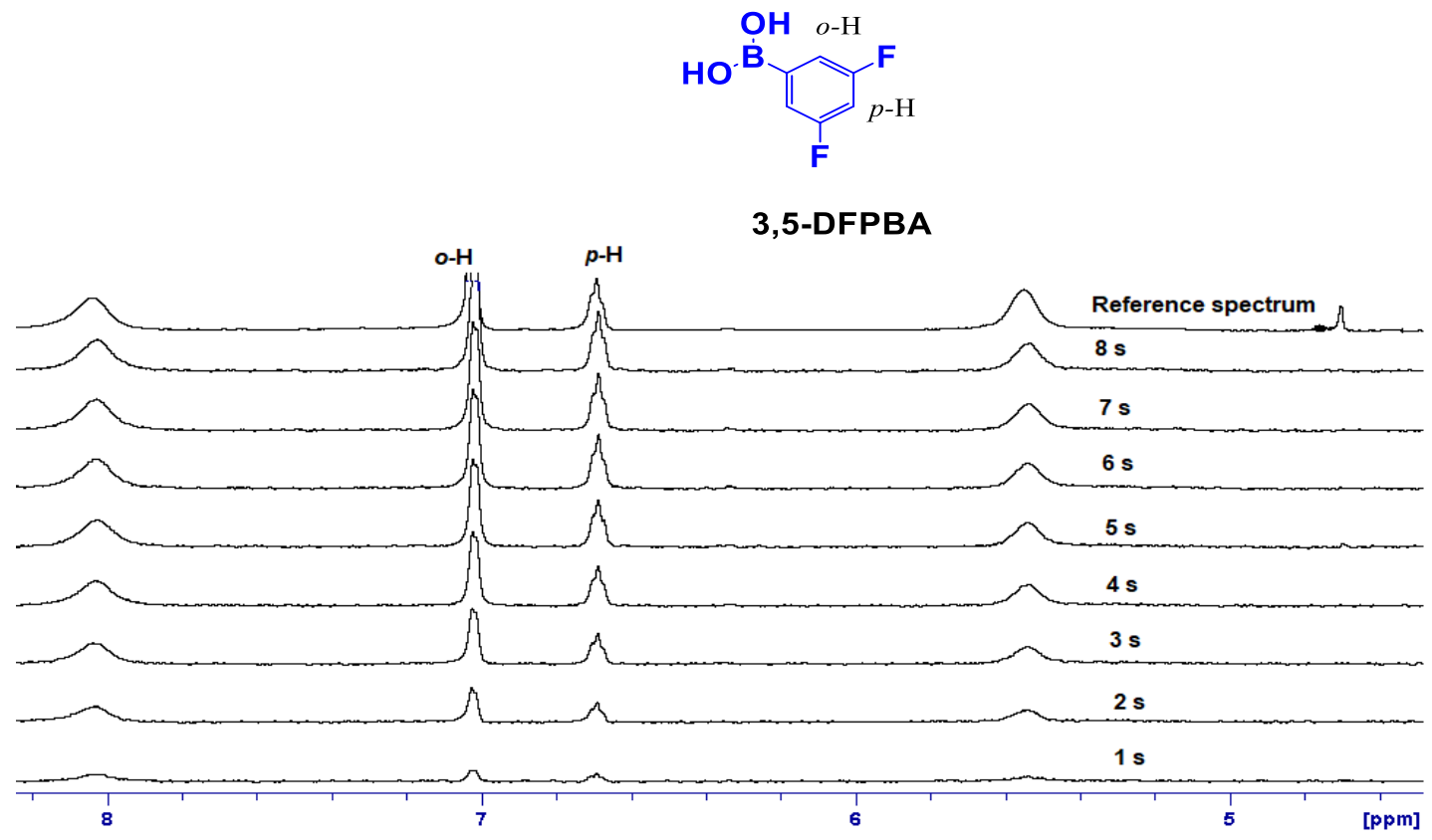

Figure 4 - Representative STD spectra of 3,5-DFPBA (1 mM) in the presence of Gal90 (100 $\mu \mathrm{M})$. Intensity of the small molecule peaks increases with increasing saturation time. The reference spectrum is scaled $x 0.5$ relative to the STD spectra. The peak at $4.79 \mathrm{ppm}$ in the reference spectrum corresponds to HOD. 
The binding strength of PBA-carbohydrate interactions is dependent on the $p \mathrm{~K}_{\mathrm{a}}$ of the PBAs, where lower $p \mathrm{~K}_{\mathrm{a}}$ values have greater binding affinities to diols than those with higher $p \mathrm{~K}_{\mathrm{a}}$ values. ${ }^{37,} 38$ The PBAs 4-fluorophenylboronic acid (4-FPBA, $p \mathrm{~K}_{\mathrm{a}}=8.77$ ) and 4methoxyphenylboronic acid (4-MPBA, $\left.p \mathrm{~K}_{\mathrm{a}}=9.30\right)$, both have higher $p \mathrm{~K}_{\mathrm{a}}$ values than 3,5-DFPBA (7.08), and should both bind to Gal90 with lower affinity than 3,5-DFBPA. ${ }^{39,40}$ Consequently, the former should exhibit lower STD-AF values than 3,5-DFPBA, while 4-MPBA would exhibit the smallest STD-AF values. STD-NMR experiments were carried out with both of these monosubstituted PBAs, and the resulting AF values bear out these predictions. The 4-FPBA STD effect builds up with increasing saturation time, eventually reaching a plateau (orange curves) with the meta proton exhibiting similar STD-AF to the ortho proton, with STD-AF 0 values of 0.48 and 0.44, respectively. 4-MPBA has the slowest buildup (red curves) and it exhibits the smallest STD

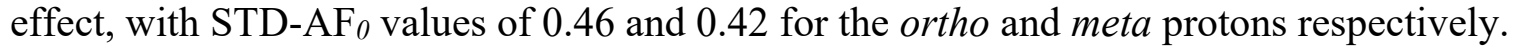

The binding affinity of PBA for carbohydrates also depends on the stereochemical relationships of the interacting hydroxyl groups on the sugar. The binding affinity of PBAs with galactose is proposed to be greater than that with glucose, as the former contains a vicinal cis-diol motif lacking in the latter. ${ }^{41}$ Therefore, we sought to explore the interactions of 3,5-DFPBA with a different sugar, glucose (Glc). The Glc90 glycopolymer was synthesized in a similar manner to Ga190 (Figure S1). We confirmed that spin diffusion occurs successfully in Glc90 by monitoring the increase in the STD effect of the triazole proton with increasing saturation time (Figure S8). Conducting the STD NMR experiment of GIc90 in the presence of 3,5-DFPBA indicates saturation transfer to protons of the 3,5-DFPBA. As expected, the STD-AF values for 3,5-DFPBA in the presence of Glc90 (Figure 5, green curves) are lower than those observed in the presence of Gal90 while, they are greater than those obtained for 4-FPBA and 4-MPBA in the presence of Gal90. 
The epitope map for 3,5-DFPBA in the presence of Glc90 indicates that para proton exhibits a larger $\mathrm{STD}-\mathrm{AF}_{0}(0.67)$ compared to the ortho proton $(0.56)$, suggesting that the former is in closer proximity to the Glc90 backbone than the latter. These experiments illustrate how STD NMR can effectively be used to detect the interaction between boronic acids and glycopolymers, and that trends in the magnitude of the STD effects with different PBAs and glycopolymer mirror the expected changes in binding affinity.<smiles>OBc1cc(O)c(F)c(F)c1</smiles>

3,5-DFPBA $p K_{a}=7.08$

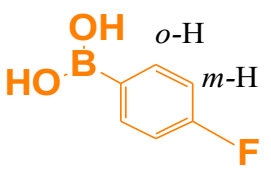

4-FPBA

$p K_{a}=8.77$<smiles>COc1ccc(B(O)O)cc1</smiles>

4-MPBA $p K_{a}=9.3$

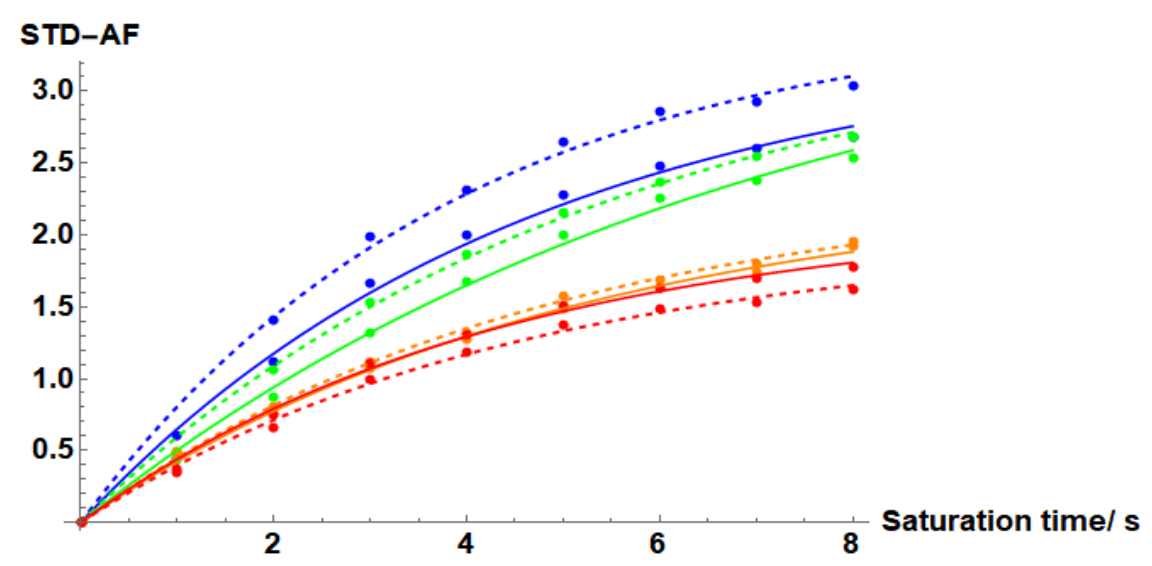

.... p-H(3,5-DFPBA + Gal90)

- O-H(3,5-DFPBA + Gal90) p-H(3,5-DFPBA + GIc90)

- O-H(3,5-DFPBA + GIC90)

$m-H(4-F P B A+G a 190)$

- O-H(4-FPBA + Gal90)

- O-H(4-MPBA + Gal90)

-... m-H(4-MPBA + Gal90)

Figure 5 - STD build up curves showing that 3,5-DFPBA shows the largest STD-AF at all saturation times in the presence of Gal90 while 4-MPBA shows the smallest STD-AF.

We used diffusion studies (DOSY) to corroborate the STD NMR data. DOSY NMR is a ligand-based NMR technique that is useful for detection of any interaction based on changes in the translational properties of the small molecules upon binding to macromolecules. ${ }^{1}$ In its free 
state, a small molecule exhibits a diffusion coefficient $(D)$ which is inversely related to the hydrodynamic radius of a molecule according to the Stokes-Einstein equation:

$$
D=\frac{k_{B} T}{6 \pi \eta r_{H}}
$$

where $\mathrm{k}_{\mathrm{B}}$ is the Boltzmann constant, $\mathrm{T}$ is temperature, $\mathrm{\eta}$ is viscosity of the solution and $\mathrm{rH}$ is the hydrodynamic radius of the solute. When the small molecule binds to a macromolecule, its effective hydrodynamic radius results in a decrease in $D$, often reported as the $\log D$ value. ${ }^{28}$ DOSY spectroscopy has been used to investigate protein-glycoconjugate systems, ${ }^{42-44}$ host-guest interactions, ${ }^{45,}, 46$ interactions of organic ligands in suspensions of nanoparticles ${ }^{47}$ as well as carbohydrate-carbohydrate interactions. ${ }^{28}$ Here, we have used this experiment to corroborate the results of the STD-NMR experiments and to illustrate the binding of the PBAs to the glycopolymers Gal90 and Glc90.

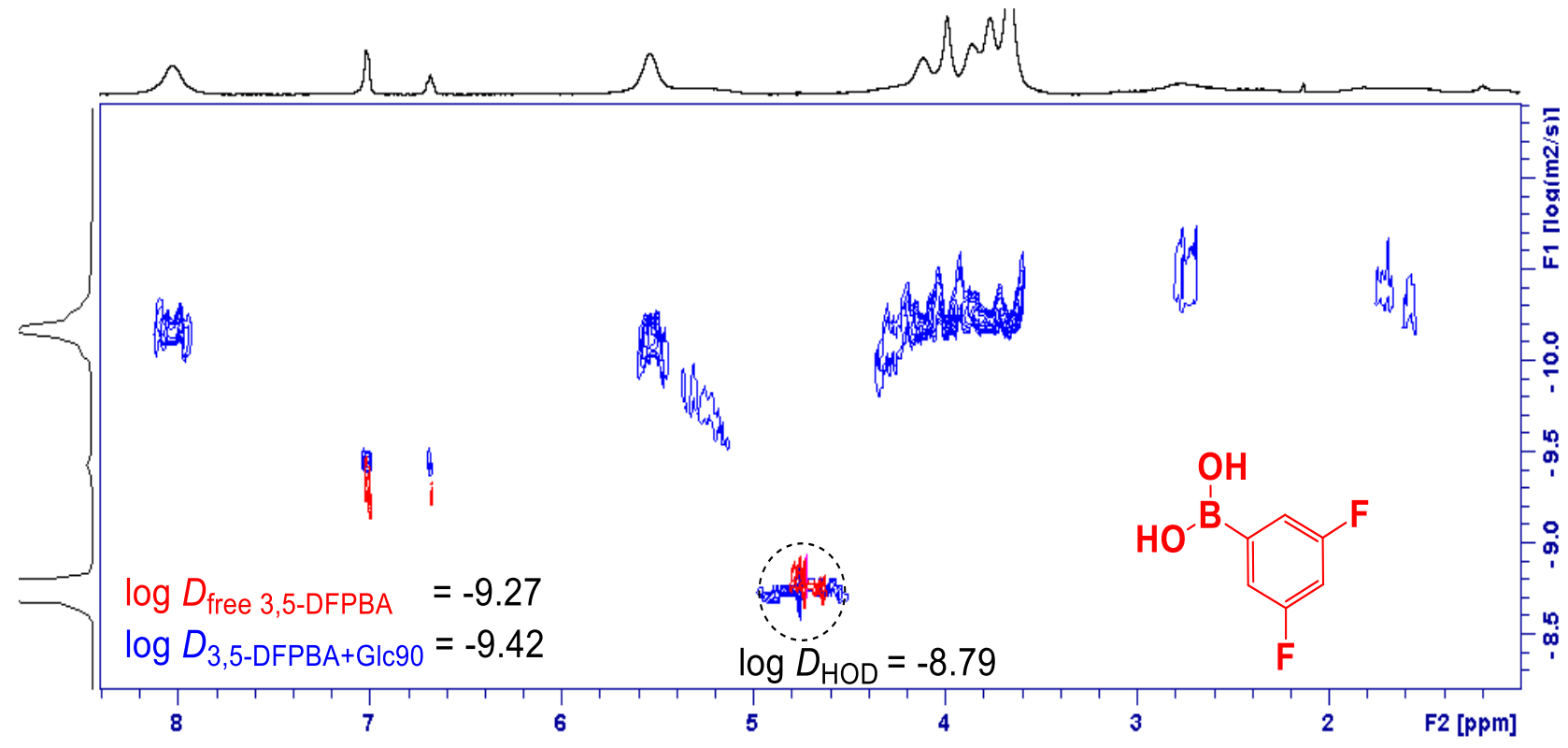

Figure 6-2D DOSY spectra. Blue - Resonances of the free 3,5-DFPBA (1 mM); Red-signals of the 3,5-DFPBA in the presence of Gal90 (EL 10 by volume). The HOD peak served as the internal reference with a $\log D$ of -8.79 . Changes in the diffusion coefficient of the 3,5-DFPBA can be seen upon addition of the Gal90. 
Figure 6 provides a representation of 2D DOSY spectra. The resonances of the free 3,5DFPBA are spread out along a horizontal line with an average $\log D$ value of -9.27 . The solvent peak seen at $4.75 \mathrm{ppm}$ is used as an internal reference with a $\log D$ of -8.79 . Upon addition of the Gal90 (excess ligand $=10$; blue resonances), the 3,5-DFPBA exhibits an average $\log D$ value of 9.42, equating to a $\Delta \log D\left(\log D_{\text {bound }}-\log D_{\text {free }}\right)$ of -0.15 . The 2D DOSY spectra of the other boronic acids in the presence or absence of Gal90 and Glc90 are shown in Figures S12-S14. Upon addition of GIc90 to 3,5-DFPBA, the $\Delta \log D$ of the PBA is -0.11. 4-FPBA exhibits a $\Delta \log D$ of 0.09 in the presence of Gal90, while the $\Delta \log D$ observed for 4-MPBA in the presence of Gal90 is -0.07 .

These $\Delta \log D$ values follow the trend previously observed with the STD technique. The 3,5-DFPBA exhibits the largest change in its $\log D$ upon addition of Gal90 while 4-FPBA exhibits the smallest change. In the presence of Gal90, the 3,5-DFPBA exhibits larger changes in its $\log D$ than in the presence of Glc90. Additional evidence for binding of 3,5-DFBA to Gal90 was provided by DOSY competition experiments in the presence of 100 -fold excess monovalent galactose (Figure 7). A $\Delta \log D$ value of -0.01 was measured upon addition of methyl- $\beta$-Dgalactopyranoside to a mixture of Gal90 and 3,5-DFPBA. The lack of an appreciable change in $\Delta \log D$ indicates that the monosaccharide inhibits the PBA from interacting with the glycopolymer by successfully competing for the boronic acid. 


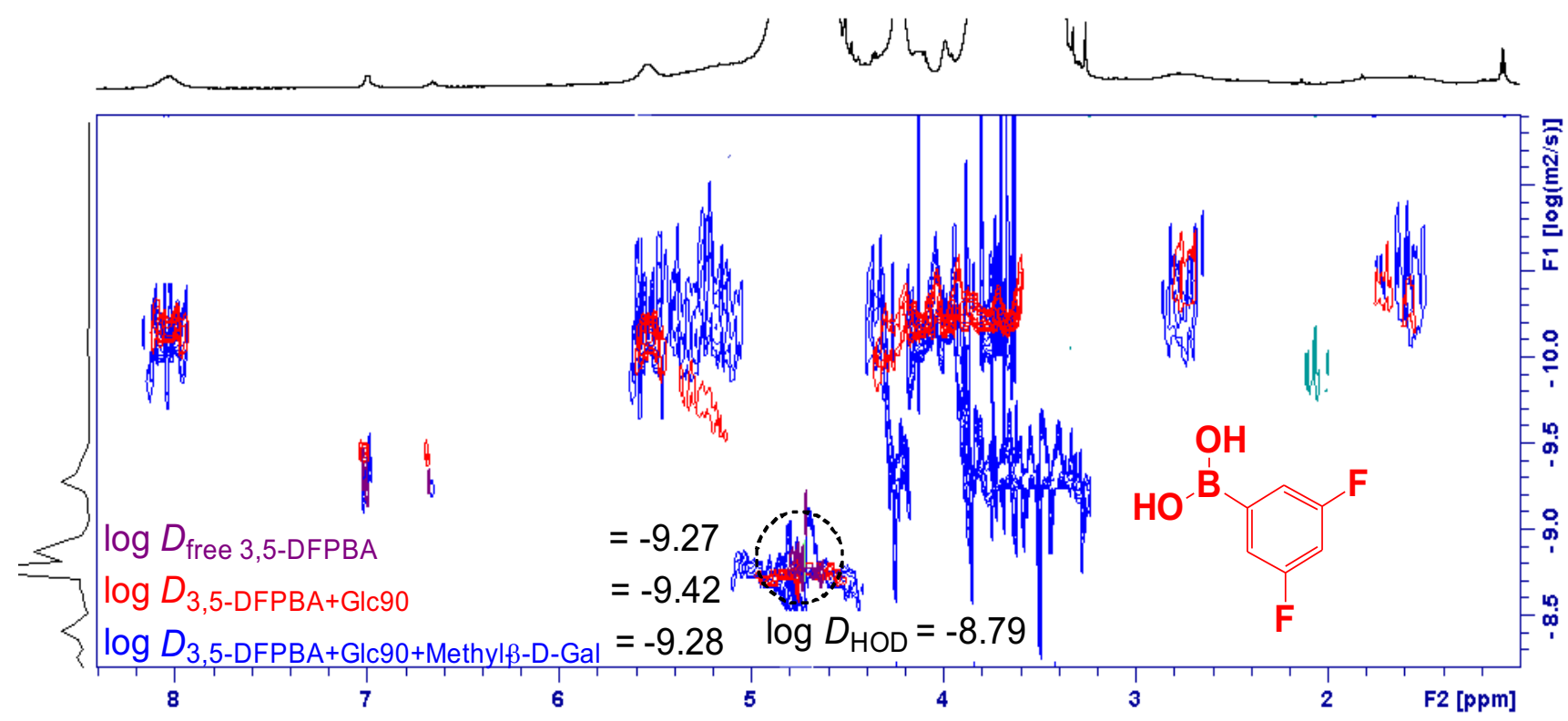

Figure 7 - DOSY competition experiment with purple signals belonging to free 3,5-DFPBA; red - 3,5DFPBA in the presence of Gal90 (EL 10) and blue - 3,5-DFPBA (290 $\mu \mathrm{M})$ in the presence of Gal90 $(29 \mu \mathrm{M})$ and methyl- $\beta$-D-Gal $(29 \mathrm{mM})$. Very small changes in the $\log D$ of the 3,5 DFPBA are observed upon addition of the monosaccharide to the 3,5-DFPBA/Gal90 mixture.

While the results above clearly demonstrate that STD NMR experiments can detect the binding of PBAs to glycopolymers, this approach can also be extended to small molecules that interact non-covalently with glycopolymers. As an illustrative example, we carried out an STDNMR experiment with Gal90 using indole as the small molecule ligand. Indole interacts with Gal via $\mathrm{C}-\mathrm{H}-\pi$ interactions. ${ }^{24,25}$ This interaction has been observed in sugar-bound protein crystal structures that indicate close proximity between ligand-derived Gal residues and the indole sidechain of tryptophan, as well as in NMR titrations of monosaccharides with indole. ${ }^{23,48,49}$ The STDAF buildup curves for the indole proton resonances as a function of saturation time are shown in Figure 8. Figure 8 indicates that indole interacts with Gal90 given that its various resonances exhibit an increase in STD-AF with increasing saturation time. Negative control experiments in the absence of Gal90 resulted in total signal cancellation of the indole resonances (Figure S16), confirming that the observed STD effect arise from transfer of saturation from the Gal90 to the 
indole. Figures 8 and Figure S18 also indicate a spatial preference for the galactose $\bullet$ indole interaction, as the amplification factors for $\mathrm{H} 2$ and $\mathrm{H} 7$ are lower than those for the other protons in indole. This experiment provides an indole-centered perspective of this interaction, which has typically been examined by examining chemical shift perturbations in the carbohydrate ${ }^{23,48,49} \mathrm{We}$ have also performed STD NMR titrations of indole with Gal90 in order to determine the binding affinity of these glycopolymer-indole interactions and this data will be presented elsewhere.

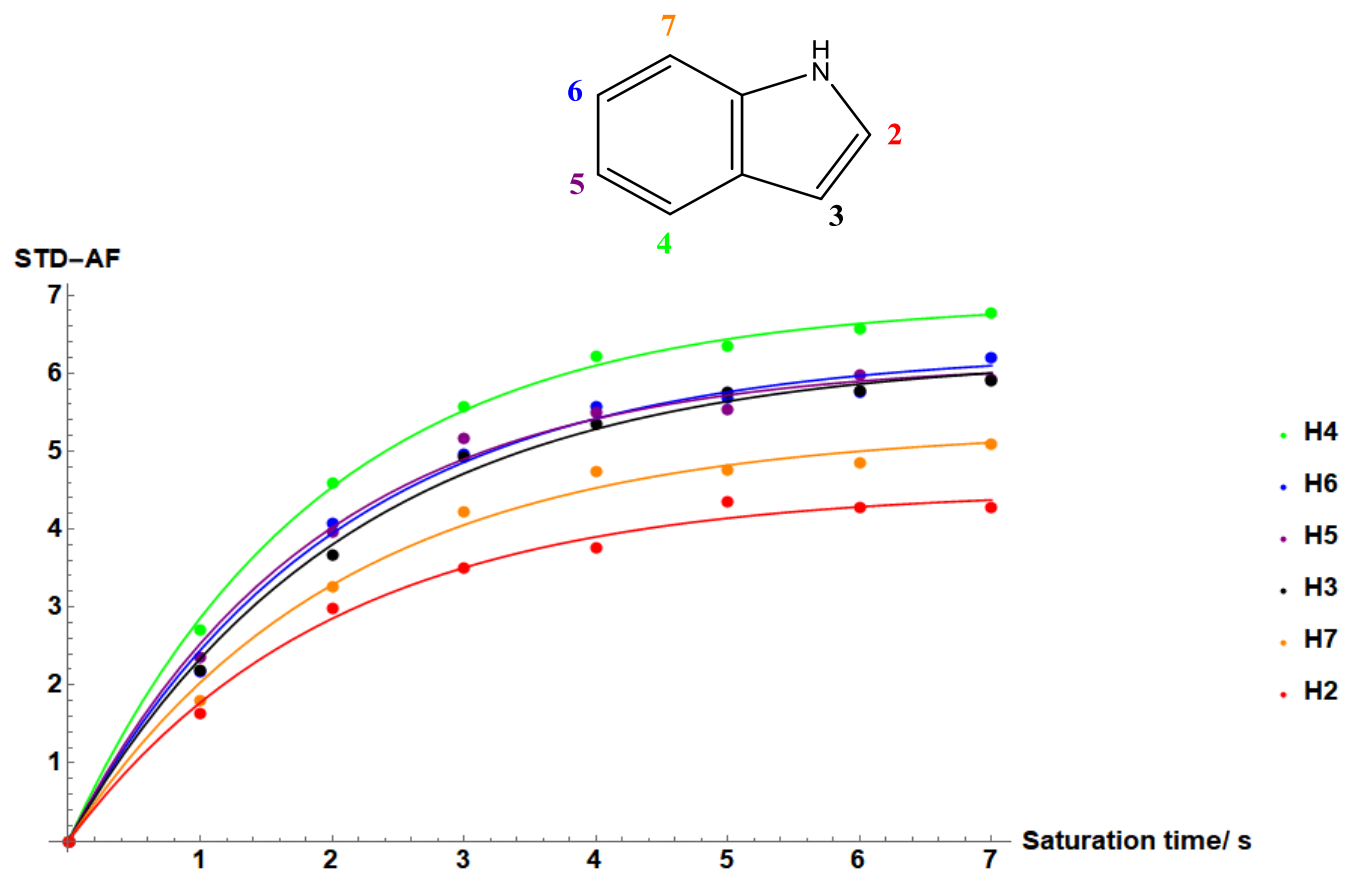

Figure 8 - STD build up curves showing that indole (3 mM) interacts with Gal90 $(50 \mu \mathrm{M})$. H4 exhibits the largest STD-AF at all saturation times while H2 exhibits the smallest STD-AF.

\section{Conclusion}

In this work, we have shown that glycopolymers can serve as the macromolecular component of an STD NMR experiment. Resonances on the polymer backbone can successfully be saturated and undergo spin diffusion. Small molecules such as PBAs or indole that interact with glycopolymers can receive this saturation from the glycopolymers and generate a saturation transfer epitope map 
that provides additional information about the orientation of the ligand with respect to the glycopolymer. The interaction of various PBAs with glycopolymers has been detected using STD NMR and DOSY NMR. This work demonstrates the use of glycopolymers in STD NMR studies to generate epitope maps for ligand-binding. The techniques described here can be applied to investigate and quantify the binding affinities of glycopolymers with other small molecules, and may be applicable to the study of other host-guest interactions in polymeric systems. ${ }^{50,51}$

\section{Acknowledgements}

This work was partially supported by the National Science Foundation (CHE1607554). Dr. Russell Hopson is thanked for his thanked for his assistance with NMR spectroscopy.

\section{References}

1. Marchetti, R.; Perez, S.; Arda, A.; Imberty, A.; Jimenez-Barbero, J.; Silipo, A.; Molinaro, A., "Rules of Engagement" of Protein-Glycoconjugate Interactions: A Molecular View Achievable by using NMR Spectroscopy and Molecular Modeling. ChemistryOpen 2016, 5 (4), 274-96.

2. Angulo, J.; Diaz, I.; Reina, J. J.; Tabarani, G.; Fieschi, F.; Rojo, J.; Nieto, P. M., Saturation transfer difference (STD) NMR spectroscopy characterization of dual binding mode of a mannose disaccharide to DC-SIGN. Chembiochem 2008, 9 (14), 2225-7.

3. Sylla, B.; Guegan, J. P.; Wieruszeski, J. M.; Nugier-Chauvin, C.; Legentil, L.; Daniellou, R.; Ferrieres, V., Probing beta-(1-->3)-D-glucans interactions with recombinant human receptors using high-resolution NMR studies. Carbohydr. Res. 2011, 346 (12), 1490-4.

4. McCullough, C.; Wang, M.; Rong, L.; Caffrey, M., Characterization of influenza hemagglutinin interactions with receptor by NMR. PLoS One 2012, 7 (7), e33958.

5. Fielding, L., NMR methods for the determination of protein-ligand dissociation constants. Prog. Nucl. Magn. Reson. Spectrosc. 2007, 51 (4), 219-242.

6. Friebolin, V.; Bayer, M. P.; Matyska, M. T.; Pesek, J. J.; Albert, K., 1H HR/MAS NMR in the suspended state: molecular recognition processes in liquid chromatography between steroids and a silica hydride-based cholesterol phase. J. Sep. Sci. 2009, 32 (10), 1722-8.

7. Friebolin, V.; Marten, S.; Albert, K., Characterization of binding affinities in a chromatographic system by suspended state HR/MAS NMR spectroscopy. Magn. Reson. Chem. 2010, 48 (2), 111-6. 
8. Suzuki, Y.; Shindo, H.; Asakura, T., Structure and Dynamic Properties of a Ti-Binding Peptide Bound to TiO2 Nanoparticles As Accessed by ${ }^{1} \mathrm{H}$ NMR Spectroscopy. J. Phys. Chem. B 2016, 120 (20), 4600-7.

9. $\quad \mathrm{Xu}, \mathrm{H}$.; Casabianca, L. B., Probing driving forces for binding between nanoparticles and amino acids by saturation-transfer difference NMR. Sci. Rep. 2020, 10 (1), 12351.

10. Zhang, Y.; Xu, H.; Parsons, A. M.; Casabianca, L. B., Examining Binding to Nanoparticle Surfaces Using Saturation Transfer Difference (STD)-NMR Spectroscopy. J. Phys. Chem. C 2017, 121 (44), 24678-24686.

11. Munoz-Garcia, J. C.; Inacio Dos Reis, R.; Taylor, R. J.; Henry, A. J.; Watts, A., NanodiscTargeted STD NMR Spectroscopy Reveals Atomic Details of Ligand Binding to Lipid Environments. Chembiochem 2018, 19 (10), 1022-1025.

12. Harris, K. A.; Shekhtman, A.; Agris, P. F., Specific RNA-protein interactions detected with saturation transfer difference NMR. RNA Biol. 2013, 10 (8), 1307-11.

13. Di Micco, S.; Bassarello, C.; Bifulco, G.; Riccio, R.; Gomez-Paloma, L., Differentialfrequency saturation transfer difference NMR spectroscopy allows the detection of different ligand-DNA binding modes. Angew. Chem. Int. Ed. Engl. 2005, 45 (2), 224-8.

14. Bristol, A. N.; Saha, J.; George, H. E.; Das, P. K.; Kemp, L. K.; Jarrett, W. L.; Rangachari, V.; Morgan, S. E., Effects of Stereochemistry and Hydrogen Bonding on Glycopolymer-Amyloid-beta Interactions. Biomacromolecules 2020, 21 (10), 4280-4293.

15. Johnson, E. C.; Feher, V. A.; Peng, J. W.; Moore, J. M.; Williamson, J. R. Application of NMR SHAPES Screening to an RNA Target. J. Am. Chem. Soc. 2003, 125 (51), 15724-15725.

16. Ishima, R., Protein-Inhibitor Interaction Studies Using NMR. Appl. NMR Spectrosc. 2015, $1,143-181$.

17. Espina-Benitez, M. B.; Randon, J.; Demesmay, C.; Dugas, V., Back to BAC: Insights into Boronate Affinity Chromatography Interaction Mechanisms. Separation \& Purification Reviews 2017, 47 (3), 214-228.

18. Zhang, X. T.; Liu, G. J.; Ning, Z. W.; Xing, G. W., Boronic acid-based chemical sensors for saccharides. Carbohydr. Res. 2017, 452, 129-148.

19. Pappin, B.; J, M.; A, T., Boron-Carbohydrate Interactions. In Carbohydrates Comprehensive Studies on Glycobiology and Glycotechnology, 2012, 10.5772/50630.

20. Nishio, M.; Umezawa, Y.; Fantini, J.; Weiss, M. S.; Chakrabarti, P., CH-pi hydrogen bonds in biological macromolecules. Phys. Chem. Chem. Phys. 2014, 16 (25), 12648-83.

21. Nishio, M., The $\mathrm{CH} /$ pi hydrogen bond in chemistry. Conformation, supramolecules, optical resolution and interactions involving carbohydrates. Phys. Chem. Chem. Phys. 2011, 13 (31), 13873-900.

22. Spiwok, V., CH/pi Interactions in Carbohydrate Recognition. Molecules 2017, 22 (7).

23. Spiwok, V.; Lipovova, P.; Skalova, T.; Buchtelova, E.; Hasek, J.; Kralova, B., Role of $\mathrm{CH} /$ pi interactions in substrate binding by Escherichia coli beta-galactosidase. Carbohydr. Res. 2004, 339 (13), 2275-80.

24. Sujatha, M. S.; Sasidhar, Y. U.; Balaji, P. V., Insights into the role of the aromatic residue in galactose-binding sites: MP2/6-311G++** study on galactose- and glucose-aromatic residue analogue complexes. Biochemistry 2005, 44 (23), 8554-62.

25. Díaz, M. D.; Fernández-Alonso, M. d. C.; Cuevas, G.; Cañada, F. J.; Jiménez-Barbero, J., On the role of aromatic-sugar interactions in the molecular recognition of carbohydrates: A 3D view by using NMR. Pure Appl. Chem. 2008, 80 (8), 1827-1835.

26. Pangborn, A. B.; Giardello, M. A.; Grubbs, R. H.; Rosen, R. K.; Timmers, F. J. Safe and 
Convenient Procedure for Solvent Purification. Organometallics 1996, 15 (5), 1518-1520.

27. Mayer, M.; Meyer, B. Group Epitope Mapping by Saturation Transfer Difference NMR to Identify Segments of a Ligand in Direct Contact with a Protein Receptor. J. Am. Chem. Soc. 2001, $123(25), 6108-6117$.

28. Santos, J. I.; Carvalho de Souza, A.; Canada, F. J.; Martin-Santamaria, S.; Kamerling, J. P.; Jimenez-Barbero, J., Assessing carbohydrate-carbohydrate interactions by NMR spectroscopy: the trisaccharide epitope from the marine sponge Microciona prolifera. Chembiochem 2009, 10 (3), 511-9.

29. Meyer, B.; Peters, T. NMR Spectroscopy Techniques for Screening and Identifying Ligand Binding to Protein Receptors. Angew. Chemie - Int. Ed. 2003, 42 (8), 864-890.

30. Kuroda, Y.; Kitamura, K. Intra-and Intermolecular ${ }^{1} \mathrm{H}-{ }^{1} \mathrm{H}$ Nuclear Overhauser Effect Studies on the Interactions of Chlorpromazine with Lecithin Vesicles. J. Am. Chem. Soc. 1984, $106(1), 1-6$.

31. Baldisseri, D; Bruker Biospin. Practical Aspects of Fragment-Based Screening Experiments in TopSpin. [Online], 2018, 3.

https://www.bruker.com/products/mr/nmr/software/fragment-based-screening-with-nmr.html (accessed October 27, 2020)

32. Ley, N. B.; Rowe, M. L.; Williamson, R. A.; Howard, M. J., Optimising selective excitation pulses to maximise saturation transfer difference NMR spectroscopy. RSC Adv. 2014, 4 (14), 7347-7351.

33. Angulo, J.; Nieto, P. M., STD-NMR: application to transient interactions between biomolecules-a quantitative approach. Eur. Biophys. J. 2011, 40 (12), 1357-69.

34. Mayer, M.; James, T. L. Detecting Ligand Binding to a Small RNA Target via Saturation Transfer Difference NMR Experiments in $\mathrm{D}_{2} \mathrm{O}$ and $\mathrm{H}_{2} \mathrm{O}$. J. Am. Chem. Soc. 2002, 124 (45), $13376-13377$.

35. Claasen, B.; Axmann, M.; Meinecke, R.; Meyer, B. Direct Observation of Ligand Binding to Membrane Proteins in Living Cells by a Saturation Transfer Double Difference (STDD) NMR Spectroscopy Method Shows a Significantly Higher Affinity of Integrin AIIb $\beta 3$ in Native Platelets than in Liposomes. J. Am. Chem. Soc. 2005, 127 (3), 916-919.

36. Claridge, T. D. W. Protein-Ligand Screening by NMR. In High-Resolution NMR Techniques in Organic Chemistry, 3rd ed.; Elsevier Science: Oxford, 2016; pp 430-437.

37. Yan, J.; Springsteen, G.; Deeter, S.; Wang, B., The relationship among pKa, pH, and binding constants in the interactions between boronic acids and diols - it is not as simple as it appears. Tetrahedron 2004, 60 (49), 11205-11209.

38. Bassil, E.; Hu, H.; Brown, P. H., Use of phenylboronic acids to investigate boron function in plants. Possible role of boron in transvacuolar cytoplasmic strands and cell-to-wall adhesion. Plant Physiol. 2004, 136 (2), 3383-95.

39. Gozdalik, J. T.; Adamczyk-Woźniak, A.; Sporzyński, A., Influence of fluorine substituents on the properties of phenylboronic compounds. Pure Appl. Chem. 2018, 90 (4), 677-702.

40. D. G. Hall, in Boronic Acids. Preparation and Applications in Organic Synthesis, Medicine and Materials, Wiley-VCH, 2011, pp. 1-109.

41. Wu, X.; Li, Z.; Chen, X. X.; Fossey, J. S.; James, T. D.; Jiang, Y. B., Selective sensing of saccharides using simple boronic acids and their aggregates. Chem Soc Rev 2013, 42 (20), 8032 8048.

42. Lucas, L. H.; Larive, C. K., Measuring ligand-protein binding using NMR diffusion experiments. Concept Magn Reson. 2004, 20 A (1), 24-41. 
43. Tanoli, N. U.; Tanoli, S. A. K.; Ferreira, A. G.; Gul, S.; Ul-Haq, Z., Evaluation of binding competition and group epitopes of acetylcholinesterase inhibitors by STD NMR, Tr-NOESY, DOSY and molecular docking: an old approach but new findings. MedChemComm 2015, 6 (10), 1882-1890.

44. Marchetti, R.; Dillon, M. J.; Burtnick, M. N.; Hubbard, M. A.; Kenfack, M. T.; Bleriot, Y.; Gauthier, C.; Brett, P. J.; AuCoin, D. P.; Lanzetta, R.; Silipo, A.; Molinaro, A., Burkholderia pseudomallei Capsular Polysaccharide Recognition by a Monoclonal Antibody Reveals Key Details toward a Biodefense Vaccine and Diagnostics against Melioidosis. ACS Chem. Biol. 2015, 10 (10), 2295-302.

45. Mayzel, O.; Cohen, Y. Diffusion Coefficients of Macrocyclic Complexes Using the PGSE NMR

Technique: Determination of Association Constants. J. Chem. Soc. Chem. Commun. 1994, 16, 1901-1902.

46. Cabaleiro-Lago, C.; Nilsson, M.; Söderman, O. Self-Diffusion NMR Studies of the HostGuest Interaction between $\beta$-Cyclodextrin and Alkyltrimethylammonium Bromide Surfactants. Langmuir 2005, 21 (25), 11637-11644.

47. Van Lokeren, L.; Maheut, G.; Ribot, F.; Escax, V.; Verbruggen, I.; Sanchez, C.; Martins, J. C.; Biesemans, M.; Willem, R., Characterization of titanium dioxide nanoparticles dispersed in organic ligand solutions by using a diffusion-ordered spectroscopy-based strategy. Chem. Eur. J. 2007, 13 (24), 6957-66.

48. Hudson, K. L.; Bartlett, G. J.; Diehl, R. C.; Agirre, J.; Gallagher, T.; Kiessling, L. L.; Woolfson, D. N., Carbohydrate-Aromatic Interactions in Proteins. J. Am. Chem. Soc. 2015, 137 (48), 15152-60.

49. Jimenez-Moreno, E.; Jimenez-Oses, G.; Gomez, A. M.; Santana, A. G.; Corzana, F.; Bastida, A.; Jimenez-Barbero, J.; Asensio, J. L., A thorough experimental study of $\mathrm{CH} / \mathrm{pi}$ interactions in water: quantitative structure-stability relationships for carbohydrate/aromatic complexes. Chem. Sci. 2015, 6 (11), 6076-6085.

50. Patra, D.; Ramesh, M.; Sahu, D.; Padhy, H.; Chu, C.-W.; Wei, K.-H.; Lin, H.-C., Synthesis and applications of a novel supramolecular polymer network with multiple H-bonded melamine pendants and uracil crosslinkers. J. Polym. Sci. A: Polym. Chem. 2012, 50 (5), 967-975. 51. Pollino, J. M.; Weck, M., Non-covalent side-chain polymers: design principles, functionalization strategies, and perspectives. Chem. Soc. Rev. 2005, 34 (3), 193-207. 


\section{Supporting Information}

\section{Saturation Transfer Difference NMR spectroscopy using glycopolymers}

Janet Muzulu and Amit Basu*

Department of Chemistry, Brown University, Providence RI, USA 02912

abasu@brown.edu

$\begin{array}{ll}\text { Table of contents } & \text { Pages }\end{array}$

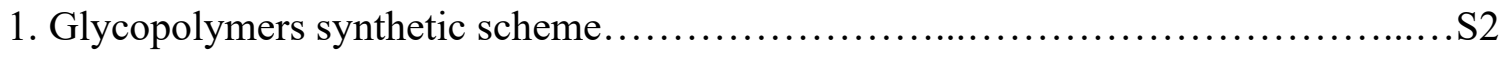

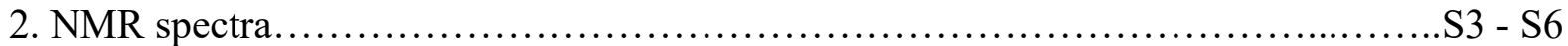

3. PBA STD NMR negative control experiments................................ 7

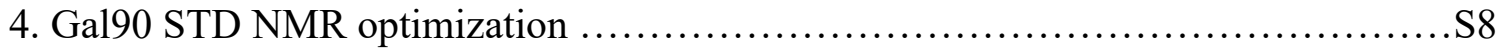

5. Glycopolymer resonances build-up curves..................................... 9

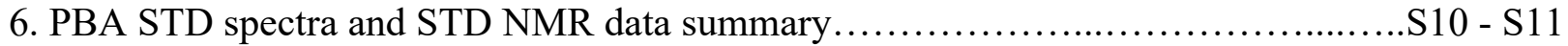

7. PBA 2D DOSY spectra and DOSY data summary............................... 12 13

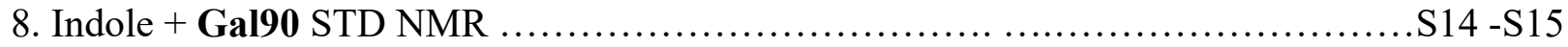

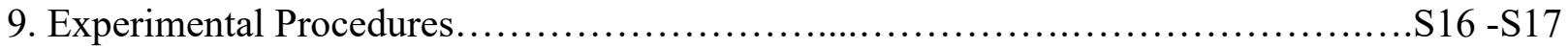

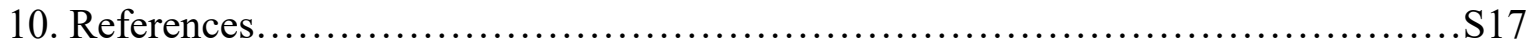




\section{Glycopolymers synthetic scheme}

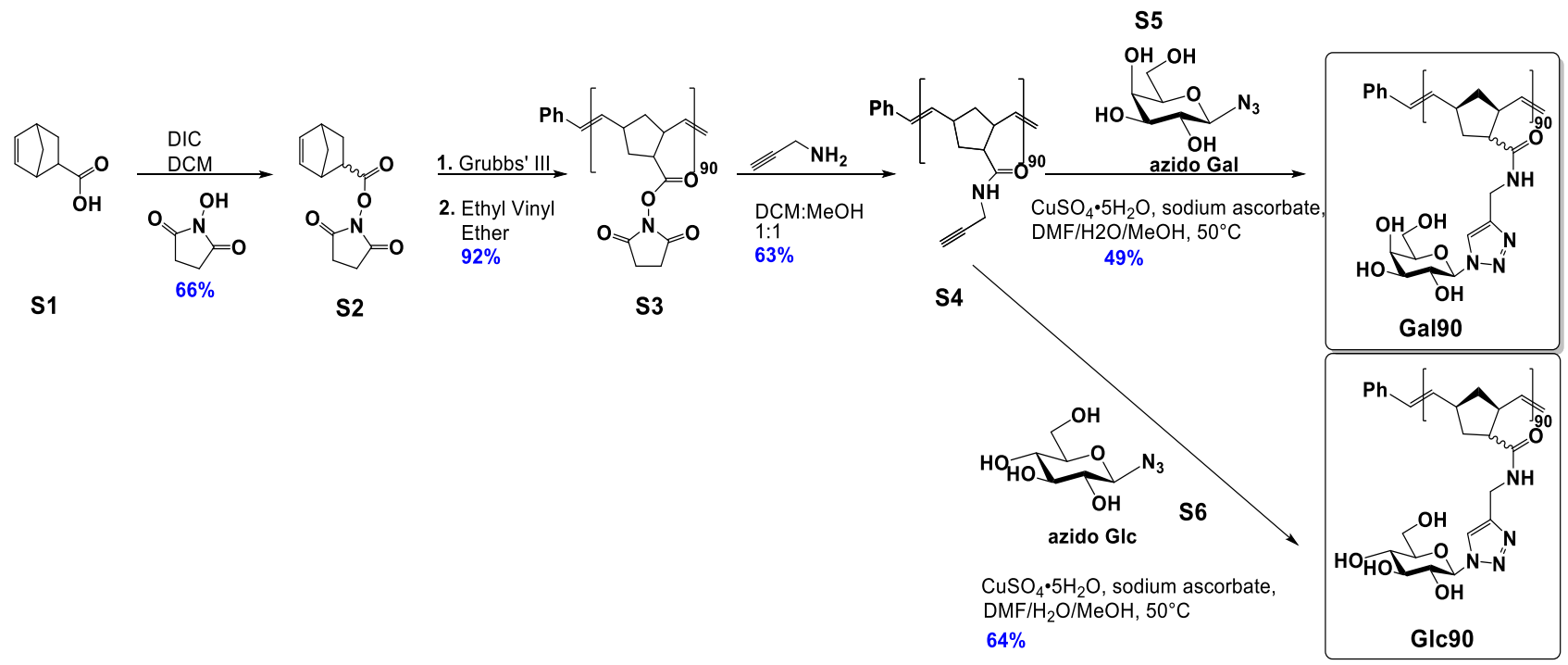

Figure S1: Reaction scheme showing the synthesis of the Gal90 and Glc90 glycopolymers 


\section{NMR spectra: Characterization}

NHS-ester ROMP Polymer (II) [ ${ }^{1} \mathrm{H}$ NMR (600 MHz, acetone-d6)]

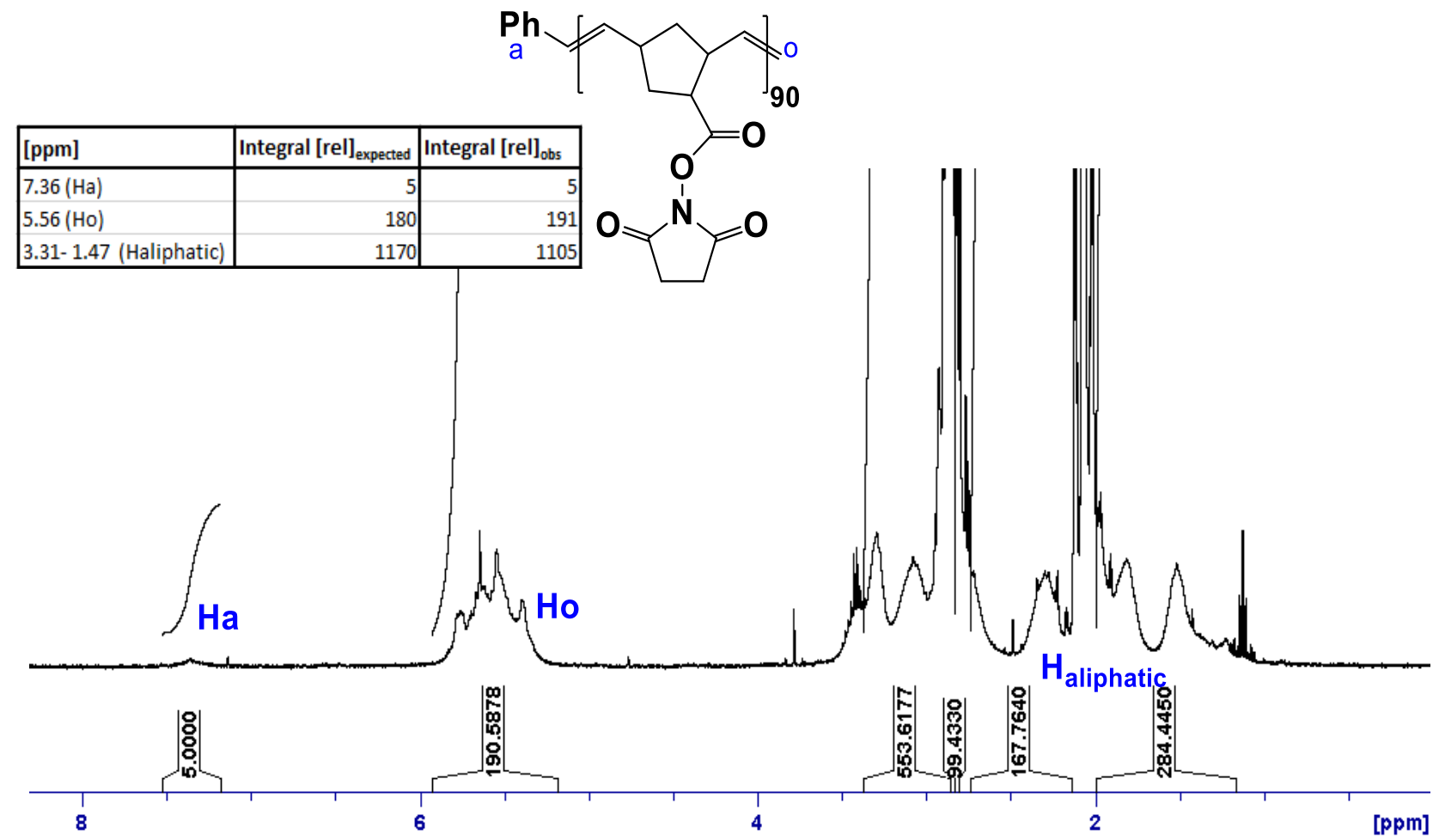

Figure S2: ${ }^{1} \mathrm{H}$ NMR spectrum of the polymer backbone in acetone- $d 6$ showing the degree of polymerization to be 95 . 


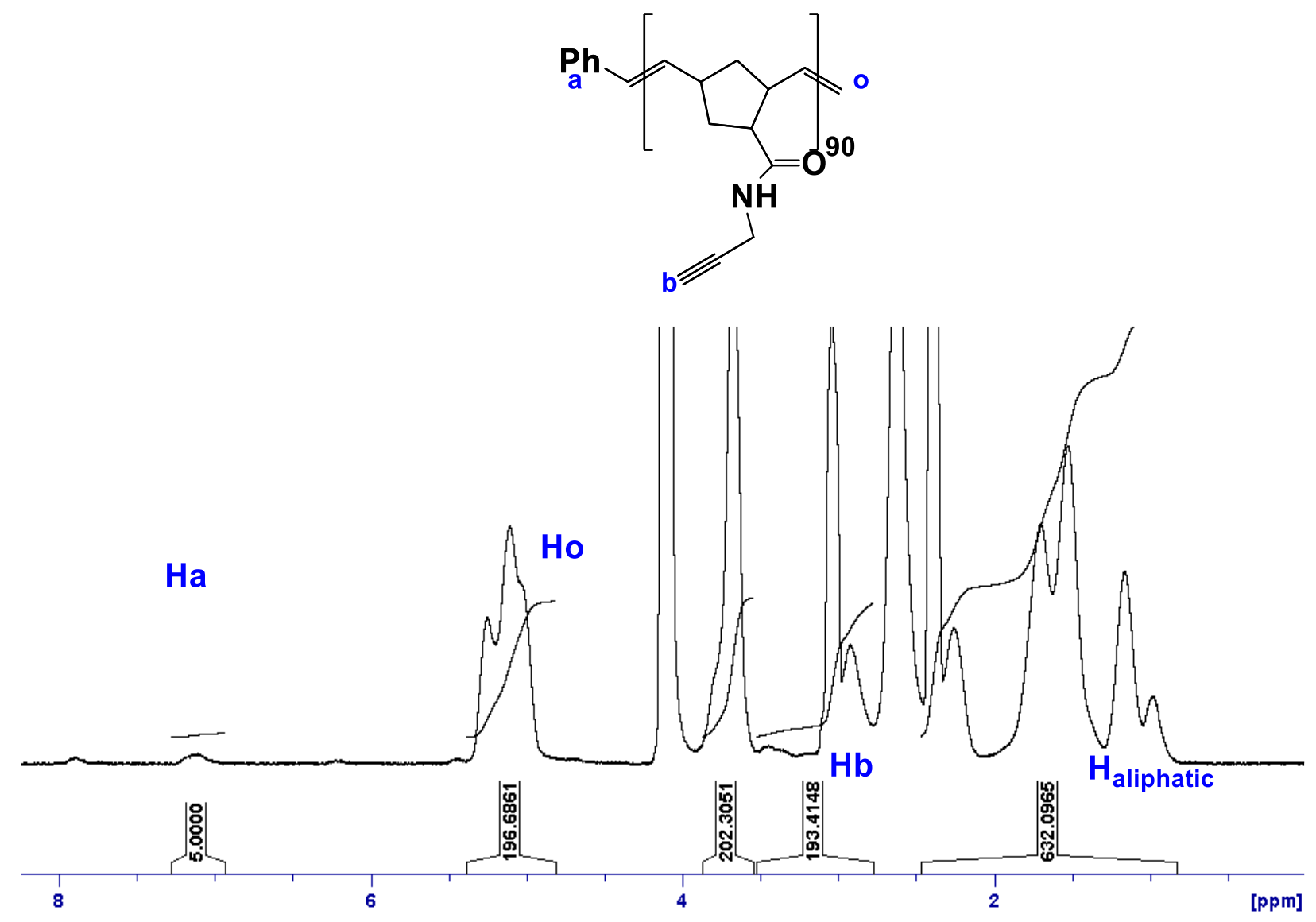

Figure S3: ${ }^{1} \mathrm{H}$ NMR of the alkyne polymer in 1:1 DMSO- $d 6 / \mathrm{CD}_{3} \mathrm{OD}$. The peak at $3.04 \mathrm{ppm}$ corresponds to the alkyne protons. 
Galactose glycopolymer (Gal90) $\left[{ }^{1} \mathrm{H}\right.$ NMR $\left.\left(600 \mathrm{MHz}, \mathrm{D}_{2} \mathrm{O}\right)\right]$

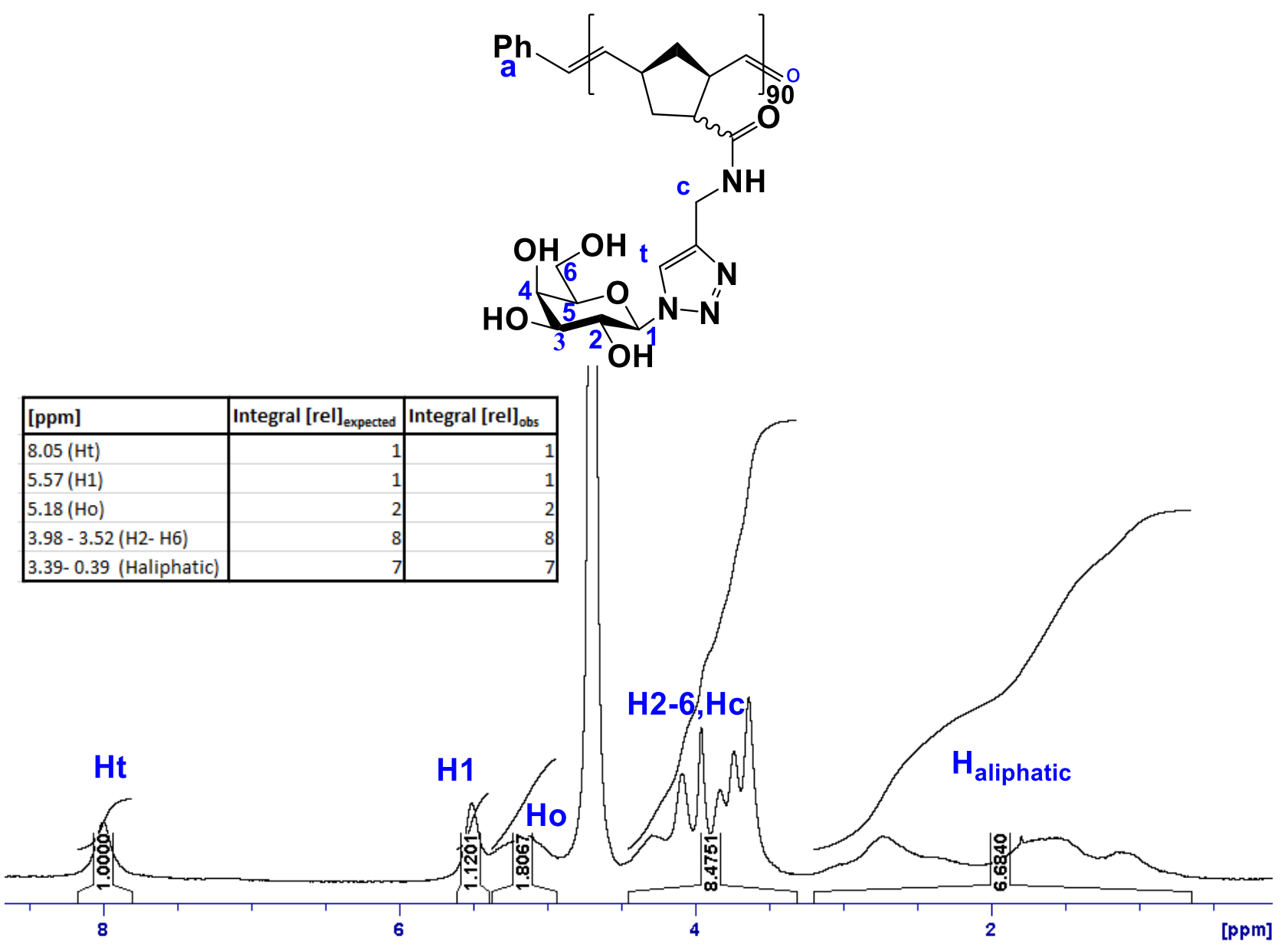

Figure S4: ${ }^{1} \mathrm{H}$ NMR of Gal90 in $\mathrm{D}_{2} \mathrm{O}$. 
Glucose glycopolymer (Glc90) $\left[{ }^{1} \mathrm{H}\right.$ NMR $\left.\left(600 \mathrm{MHz}, \mathrm{D}_{2} \mathrm{O}\right)\right]$

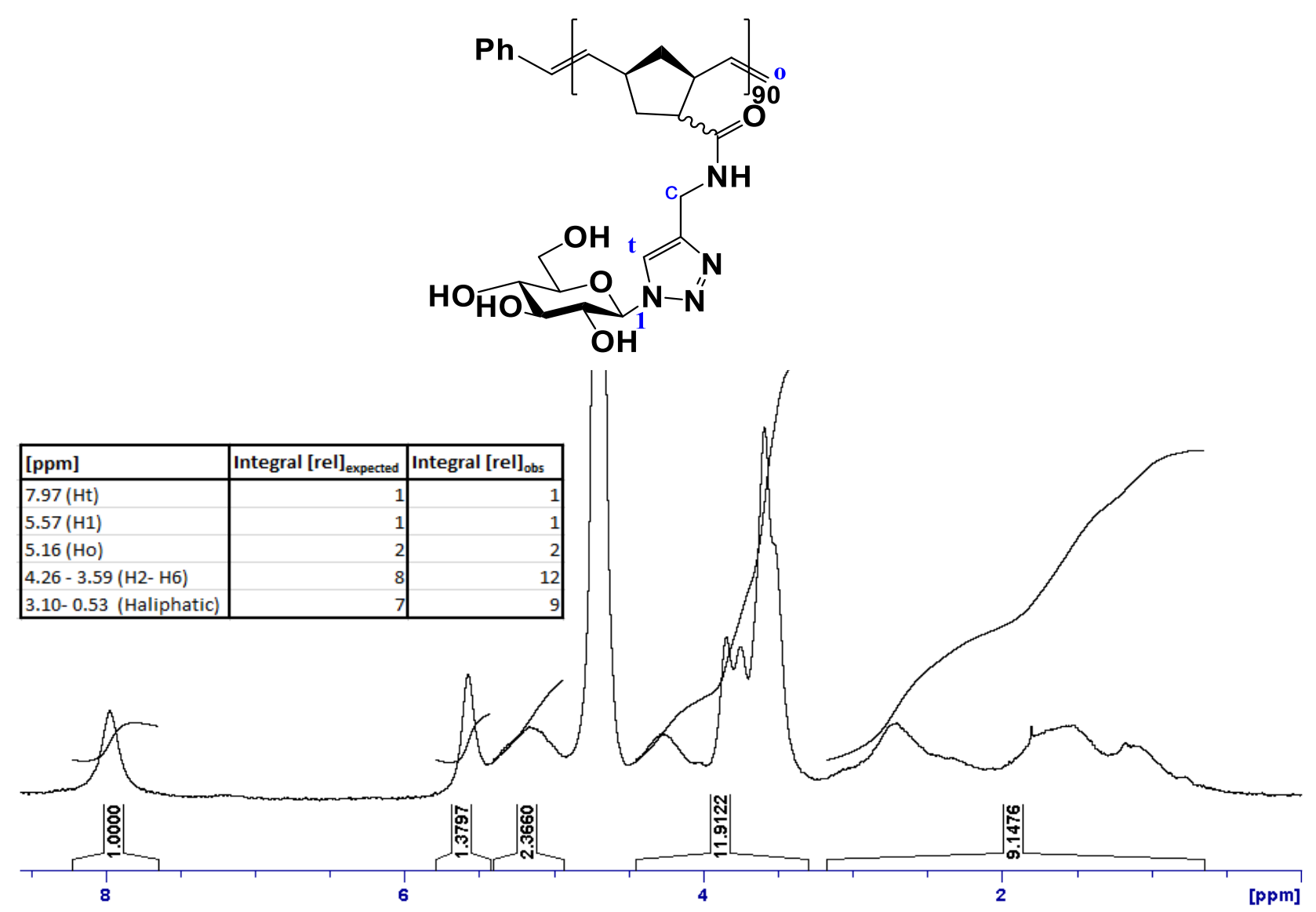

Figure S5: ${ }^{1} \mathrm{H}$ NMR of Glc90 in $\mathrm{D}_{2} \mathrm{O}$. 


\section{PBA STD NMR negative control experiments}

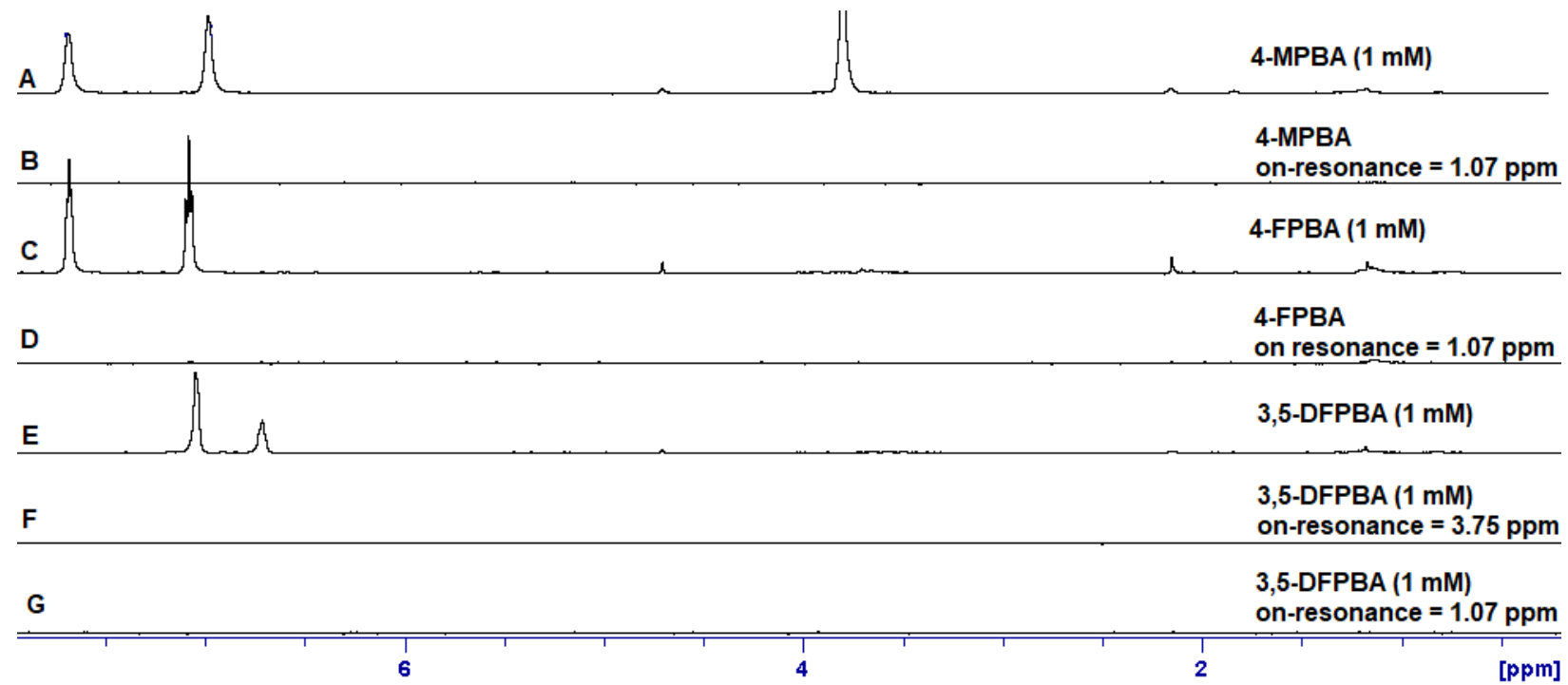

Figure S6: STD NMR negative control experiments. Reference spectra of 1mM 4-MPBA, 4FPBA and 3,5-DFPBA respectively (A, C \&E). Corresponding STD spectra of the PBAs (B, D, $\mathrm{F} \& \mathrm{G})$. The difference spectra show no small molecule resonances as there is no macromolecule to receive saturation from. All spectra were acquired using optimal parameters which included a $25 \mathrm{~ms}$ Gaus shaped pulse with a pulse power of $-47.5 \mathrm{~dB}$ at a saturation time of $1 \mathrm{~s}$. 


\section{Gal90 STD NMR optimization}

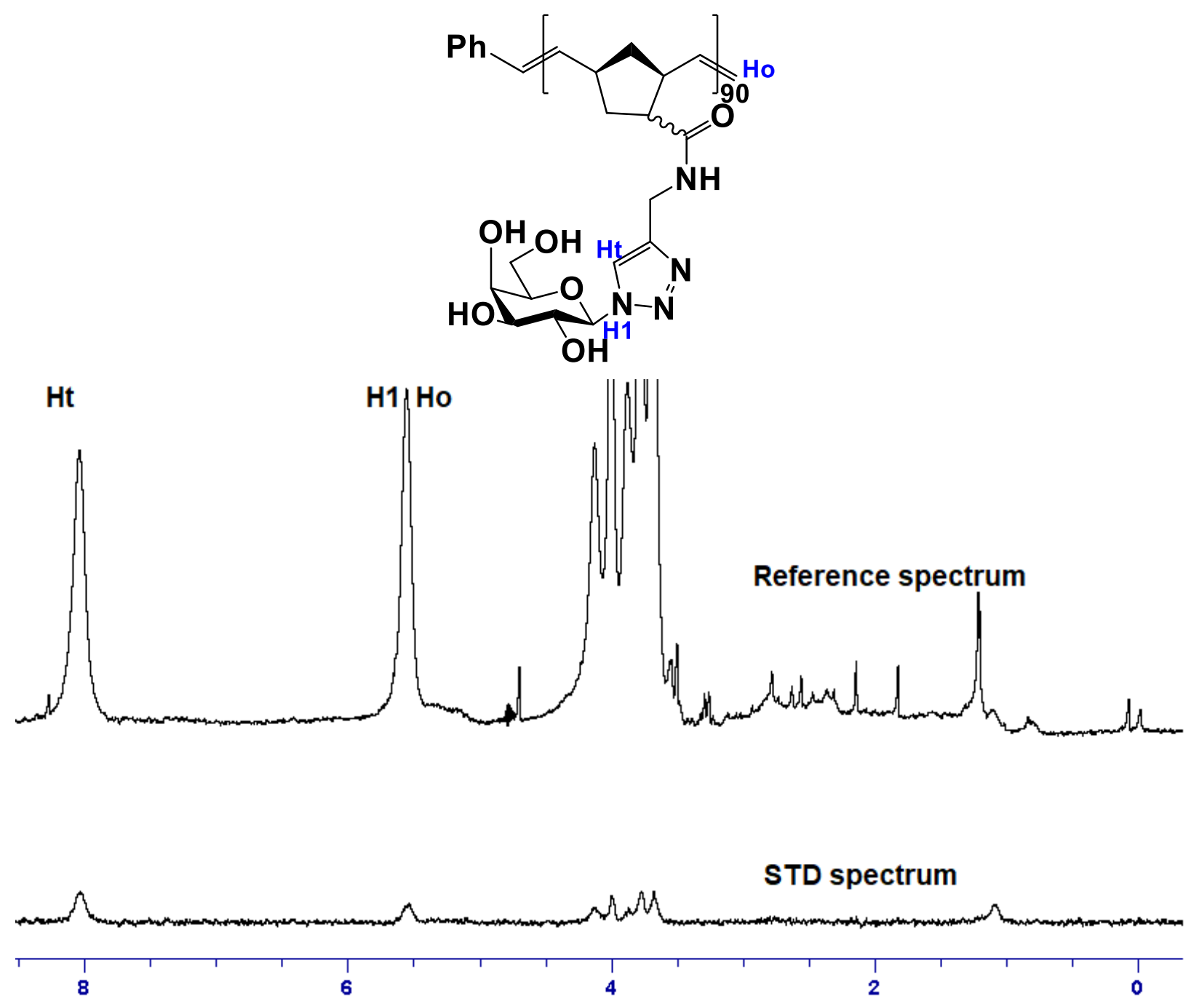

Figure S7: Gal90 spectra: Reference and STD spectra obtained using optimal parameters (bottom) (Entry 11 in text) 


\section{Glycopolymer resonances build-up curves}

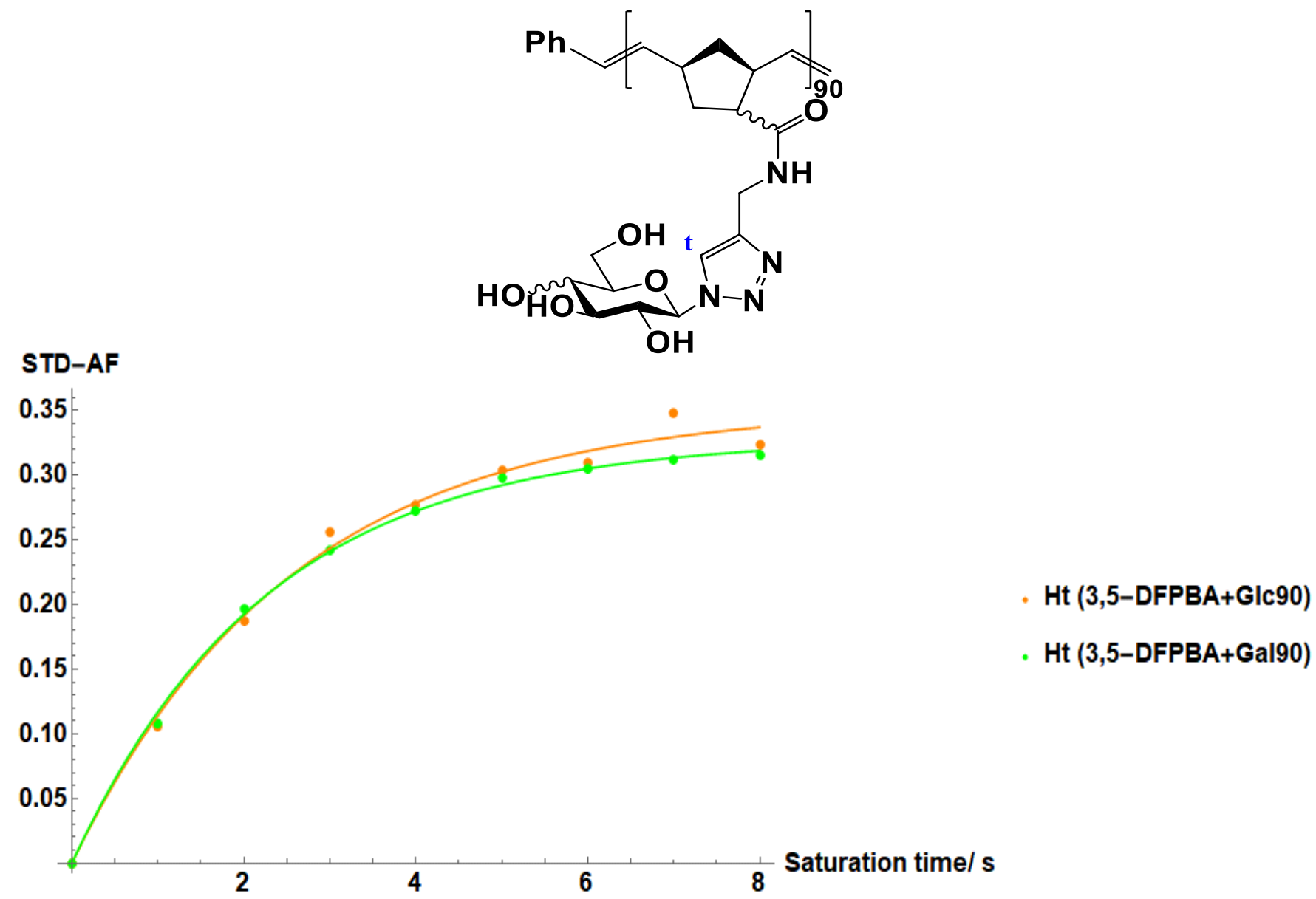

Figure S8: Build-up curves of the triazole proton (Ht) of Gal90 (green) and Glc90 (orange) in the presence of $1 \mathrm{mM} 3,5-\mathrm{DFPBA}$. The STD effect increases with increasing saturation time. 


\section{PBA STD spectra and STD NMR data summary}

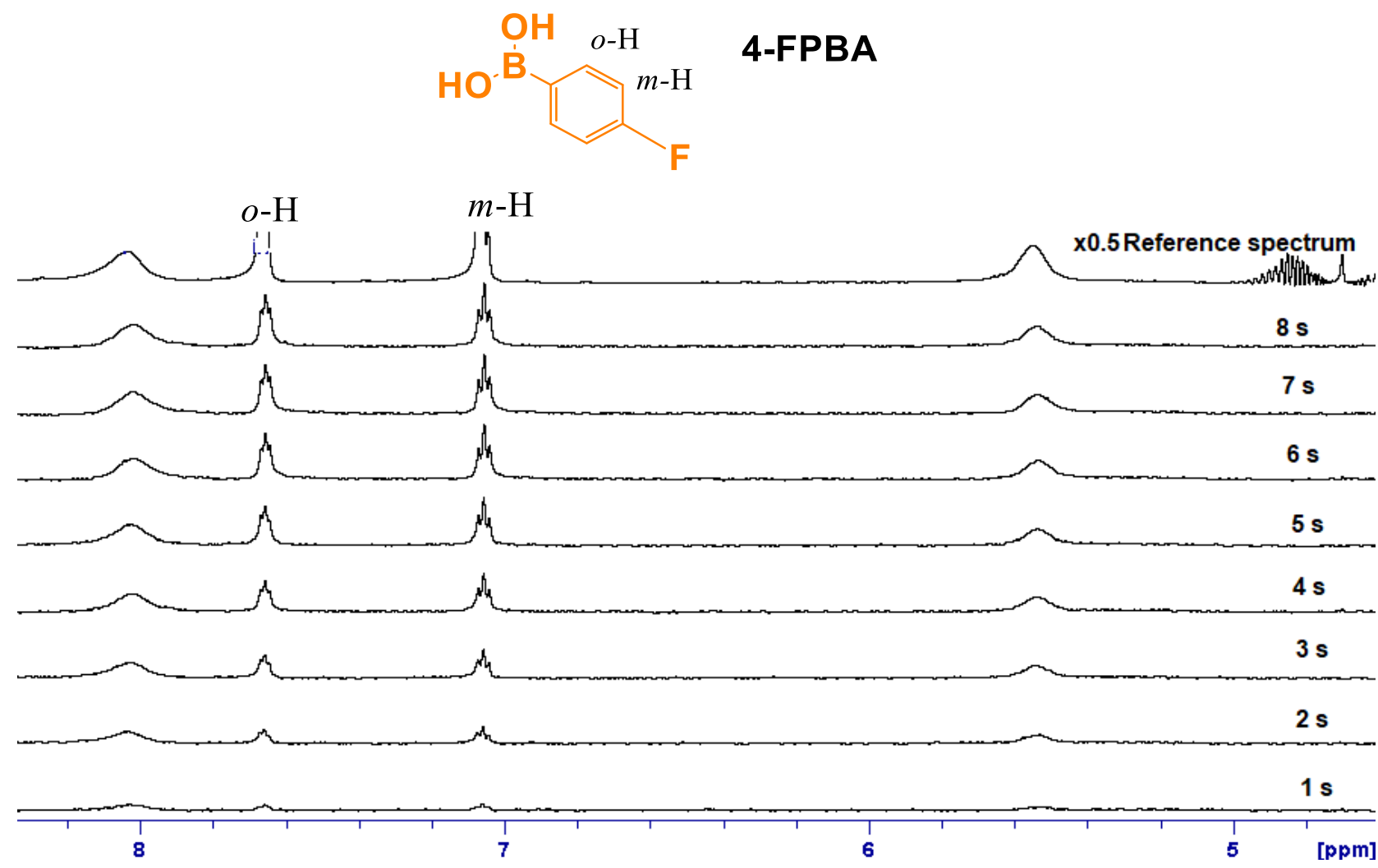

Figure S9: STD spectra of 4-FPBA $(1 \mathrm{mM})$ in the presence of Gal90 $(100 \mu \mathrm{M})$ in $\mathrm{D}_{2} \mathrm{O}$. Intensity of the small molecule peaks increases with increasing saturation time. The reference spectrum is scaled $\mathrm{x} 0.5$ 


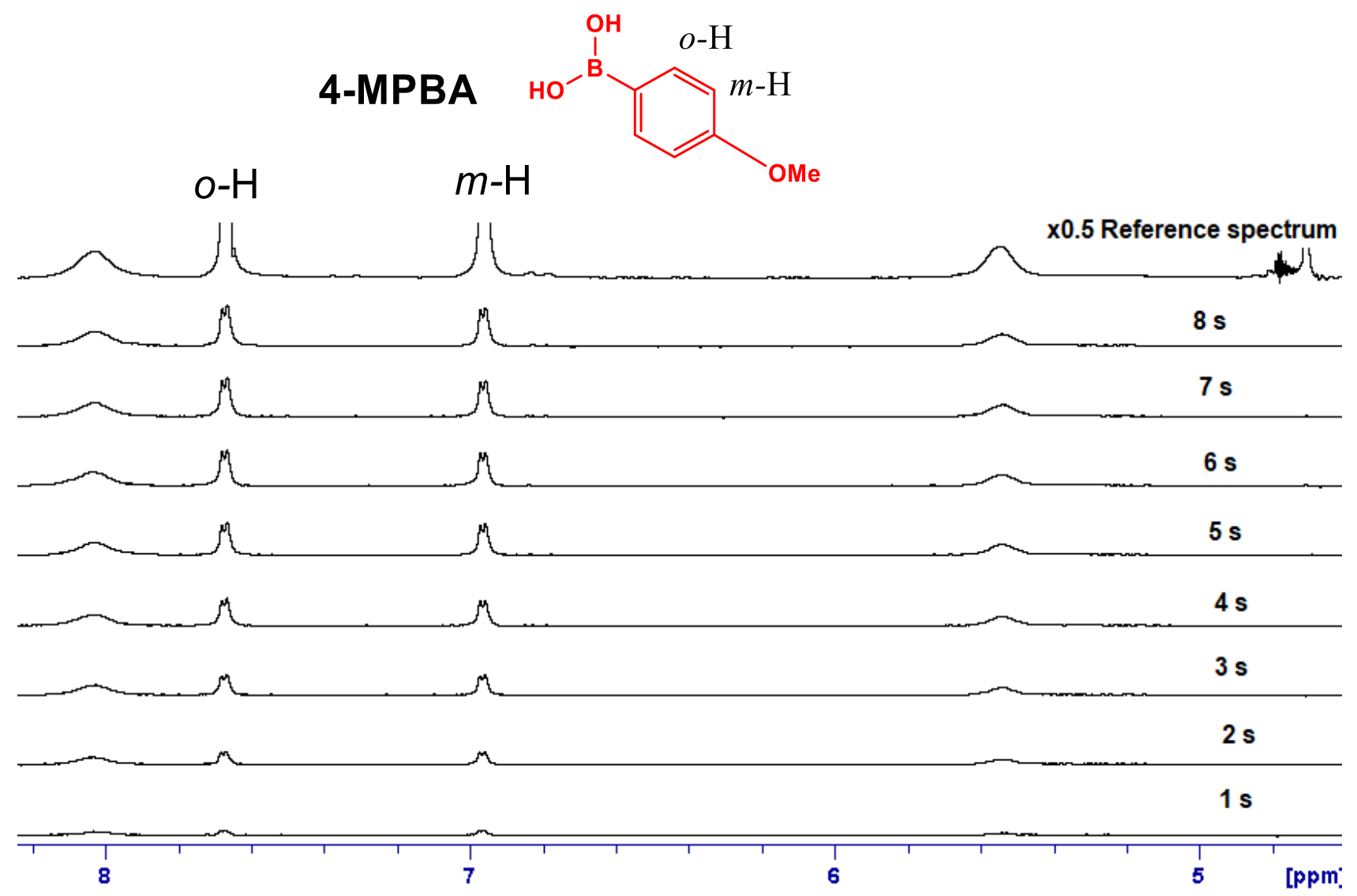

Figure S10: STD spectra of 4-MPBA $(1 \mathrm{mM})$ in the presence of Gal90 $(100 \mu \mathrm{M})$ in $\mathrm{D}_{2} \mathrm{O}$. Intensity of the small molecule peaks increases with increasing saturation time. The reference spectrum is scaled $\mathrm{x} 0.5$

\begin{tabular}{|l|l|l|r|}
\hline Entry & PBA Proton & STD-AF $_{\mathbf{0}}$ & \% amplification \\
\hline 3,5-DFPBA/Ga190 & $o-\mathrm{H}$ & $0.69 \pm 0.04$ & 78 \\
& $p-\mathrm{H}$ & $0.88 \pm 0.04$ & 100 \\
\cline { 2 - 4 } 3,5-DFPBA/Glc90 & $o-\mathrm{H}$ & $0.56 \pm 0.03$ & 84 \\
& $p-\mathrm{H}$ & $0.67 \pm 0.02$ & 100 \\
\cline { 2 - 4 } 4FPBA/Ga190 & $o-\mathrm{H}$ & $0.44 \pm 0.04$ & 93 \\
& $p-\mathrm{H}$ & $0.48 \pm 0.03$ & 100 \\
\cline { 2 - 4 } 4MPBA/Gal90 & $\mathrm{o}-\mathrm{H}$ & $0.46 \pm 0.03$ & 100 \\
\hline
\end{tabular}

Table S11: STD NMR data summary with binding epitope maps of each small molecule 


\section{PBA 2D DOSY spectra and DOSY data summary}

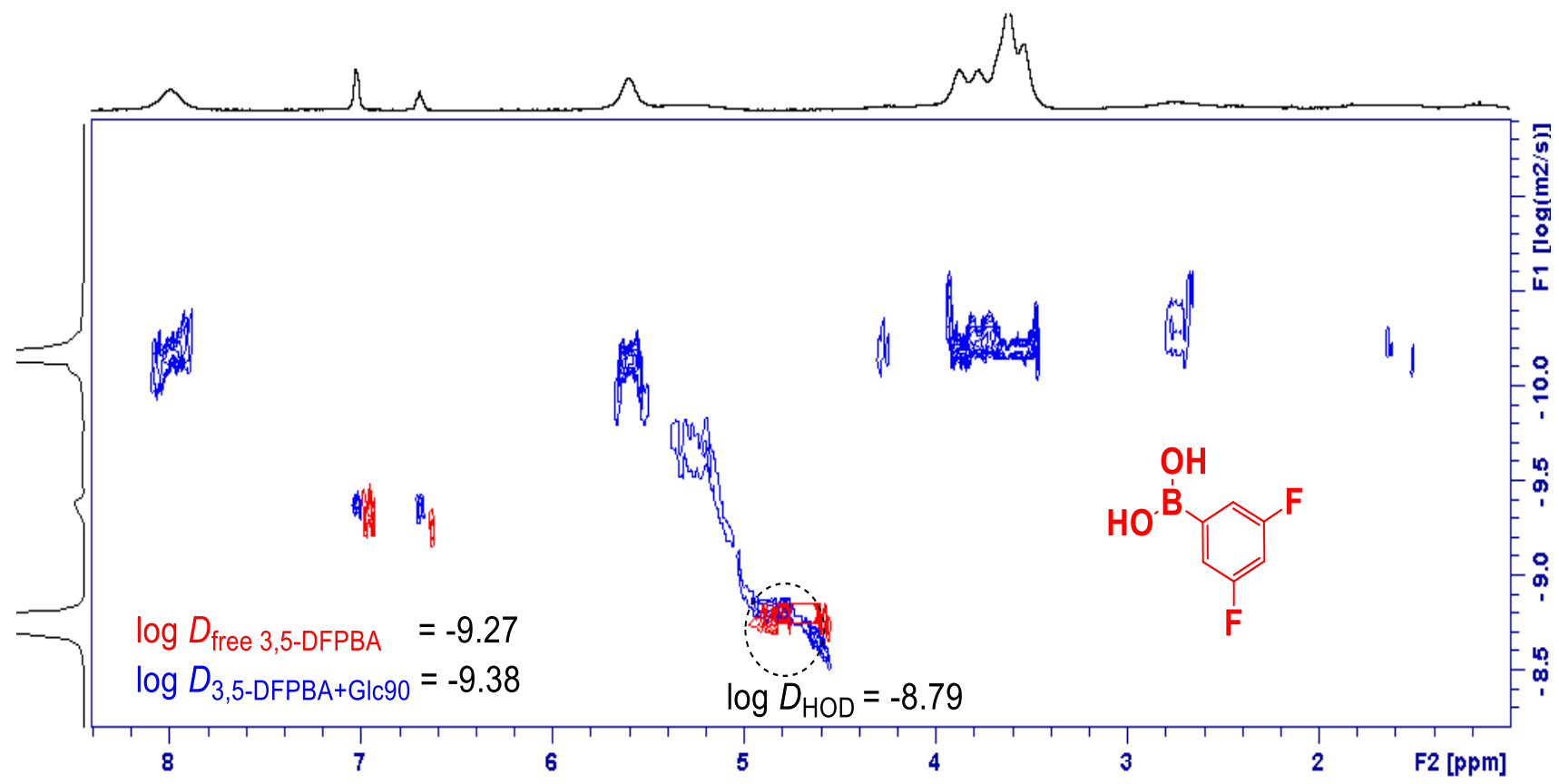

Figure S12: DOSY 2D spectrum of (red): free 4-FPBA (1 mM) and (blue): 4-FPBA (1 mM) in the presence of $\mathbf{G a l 9 0}(100 \mu \mathrm{M})$ in phosphate buffer $(0.1 \mathrm{M})$ in $\mathrm{D}_{2} \mathrm{O}, \mathrm{pH} 7.23$ at room temperature.

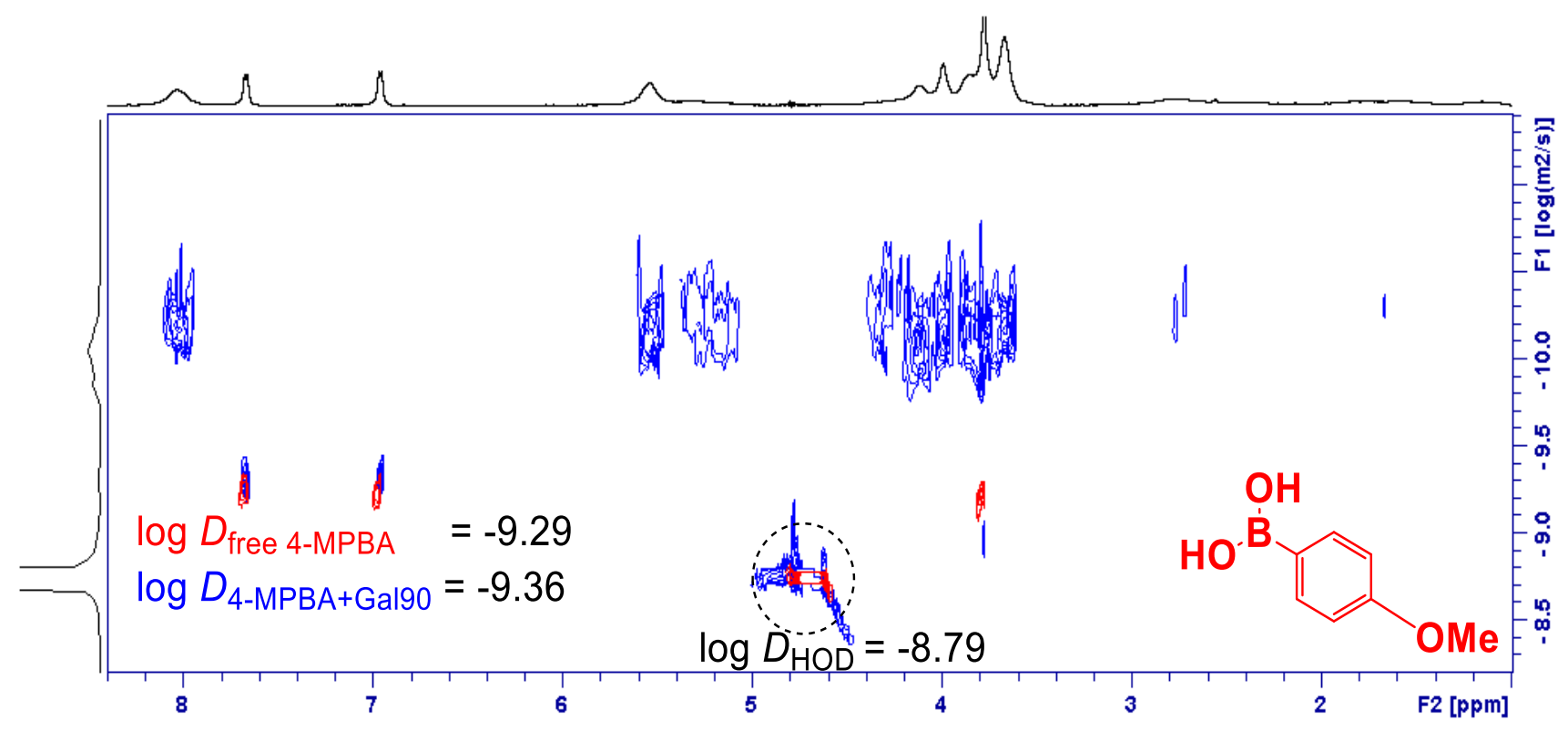

Figure S13: DOSY 2D spectrum of (red): free 4-MPBA (1 mM) and (blue): 4-MPBA (1 mM) in the presence of Gal90 $(100 \mu \mathrm{M})$ in phosphate buffer $(0.1 \mathrm{M})$ in $\mathrm{D}_{2} \mathrm{O}, \mathrm{pH} 7.23$ at room temperature. 


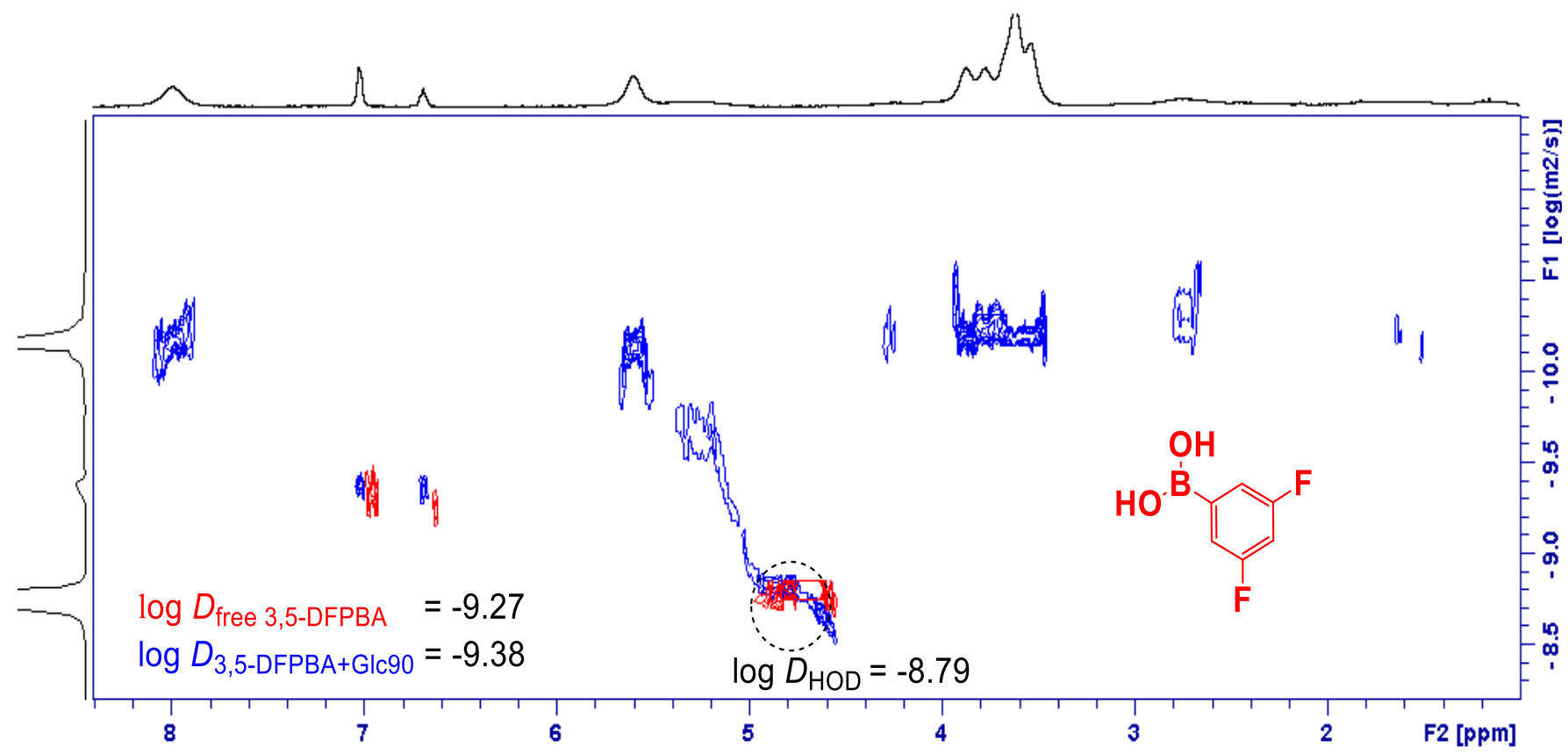

Figure S14: DOSY 2D spectrum of (red): free 3,5-DFPBA (1 mM) and (blue): 3,5-DFPBA (1 $\mathrm{mM})$ in the presence of Glc90 $(100 \mu \mathrm{M})$ in phosphate buffer $(0.1 \mathrm{M})$ in $\mathrm{D}_{2} \mathrm{O}$, pH 7.23 at room temperature.

\begin{tabular}{|l|r|r|l|}
\hline Entry & $\log \boldsymbol{D}_{\text {free PBA }}$ & $\log \boldsymbol{D}_{\text {avg mixture }}$ & $\boldsymbol{\Delta}$ \\
\hline 3,5-DFPBA/Gal90 & -9.27 & -9.42 & $-0.15 \pm 0.005$ \\
\hline 3,5-DFPBA/Glc90 & -9.27 & -9.38 & $-0.11 \pm 0.006$ \\
\hline 4FPBA/Gal90 & -9.26 & -9.35 & $-0.09 \pm 0.004$ \\
\hline 4MPBA/Ga190 & -9.29 & -9.36 & $-0.07 \pm 0.02$ \\
\hline
\end{tabular}

Figure S15: DOSY data summary showing changes in the $\log \mathrm{D}$ of the small molecules upon addition of glycopolymers (Gal90/Glc90) 


\section{Indole + Gal90 STD NMR}
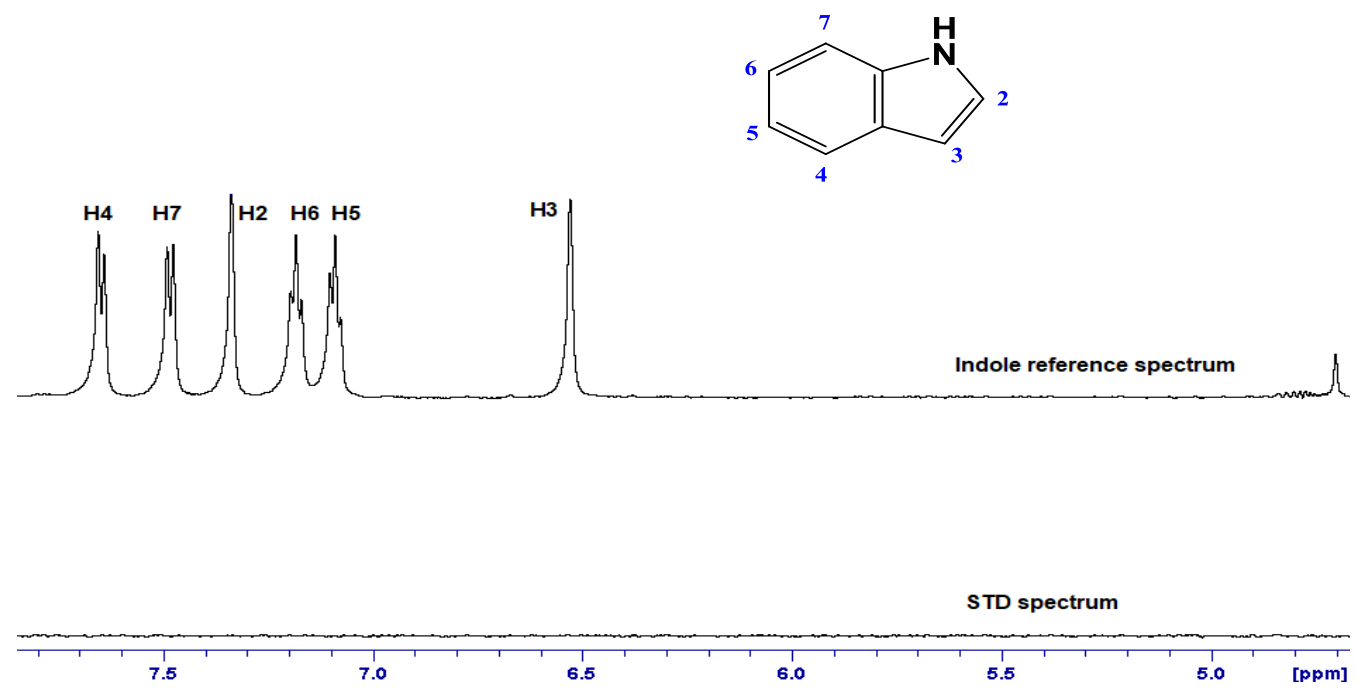

Figure S16: STD negative control experiment. Indole $(3 \mathrm{mM})$ experiences total signal cancellation in the absence of Gal90.
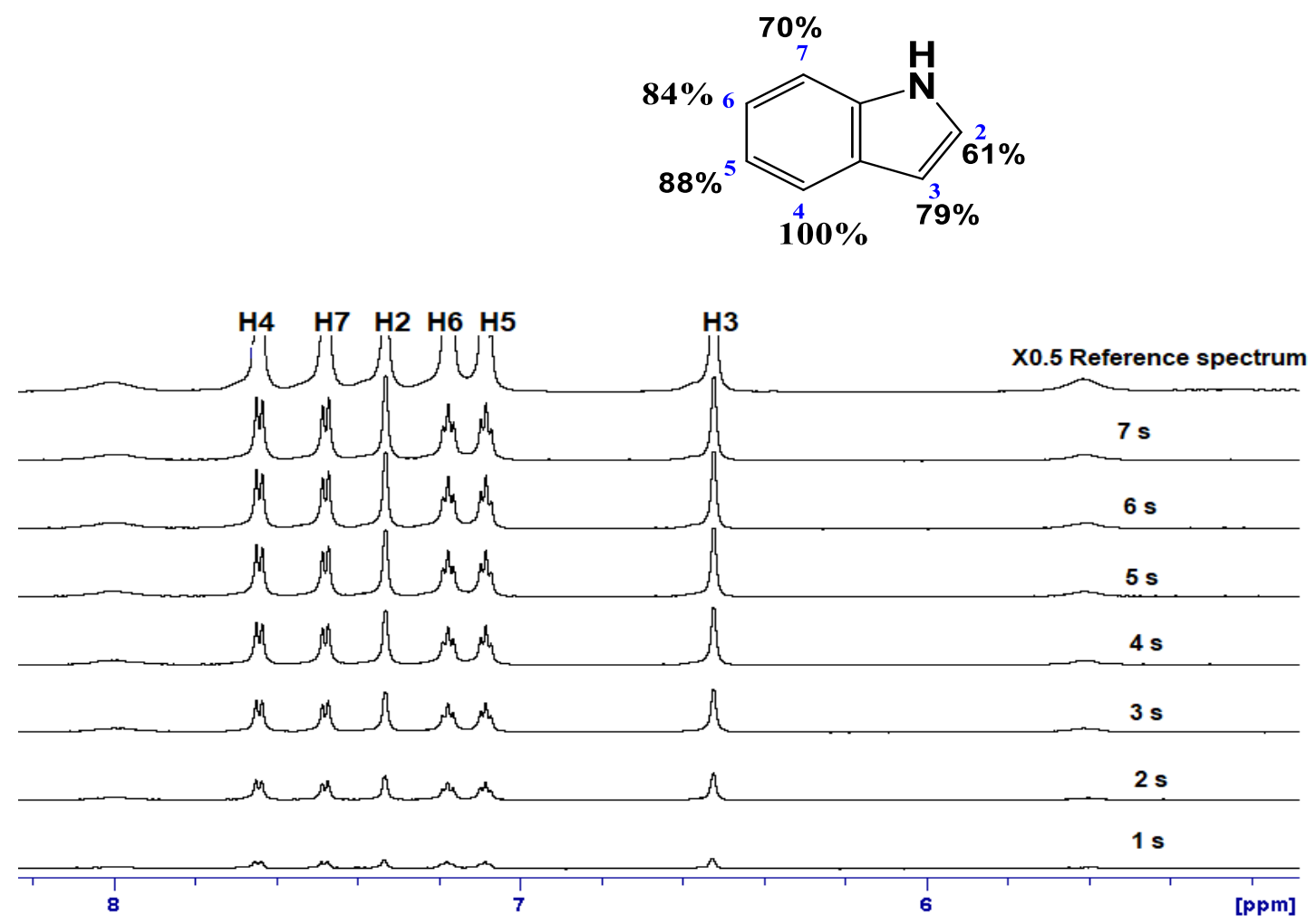
Figure S17: STD spectra of indole (3 mM) in the presence of Gal90 $(50 \mu \mathrm{M})$. Intensity of the indole resonances increases with increasing saturation time. The reference spectrum is scaled $\mathrm{x} 0.5$. The binding epitope map is shown as an insert.

\begin{tabular}{|l|r|r|}
\hline Proton & STD-AF $_{\mathbf{0}}$ & \% amplification \\
\hline H4 & 3.69 & 100 \\
H7 & 2.57 & 70 \\
H2 & 2.26 & 61 \\
H6 & 3.1 & 84 \\
H5 & 3.26 & 88 \\
H3 & 2.93 & 79 \\
\hline
\end{tabular}

Figure S18: Indole ( $3 \mathrm{mM})$ binding epitope map and $\mathrm{STD}-\mathrm{AF}_{0}$ values obtained in the presence of Gal90 $(50 \mu \mathrm{M})$ 


\section{Experimental Procedures}

Synthesis of S2: S2 was prepared as reported by Okoth et al ${ }^{l}$ and was obtained as a white solid. Yield 66\%; ' ${ }^{\mathrm{H}} \mathrm{NMR}\left(400 \mathrm{MHz}, \mathrm{CDCl}_{3}\right) \delta$ 6.27-6.09 (m, 2H, Halkene) 3.40 (s, 1H) 3.24 (dt, $J=$ 9.1, 3.8 Hz, 1H) 2.99 (s, 1H) 2.81 (bs, 4H, HNHs) 2.51 (ddd, $J=9.0,4.6,1.4 \mathrm{~Hz}, 1 \mathrm{H}) 2.09-1.93$ (m, 1H) $1.56-1.23(\mathrm{~m}, 3 \mathrm{H})$.

Synthesis of S3: S3 was prepared by a slight variation of the protocol reported by Okoth $e t$ al ${ }^{1}$ and was obtained as a gray solid. Yield 92\%; 'H NMR (600 MHz, acetone-d6) $\delta$ 7.36-7.29 (m, 5H, H-Ph) 5.39-5.76 (m, 191 H, H-olefinic) 1.12-3.42 (m, 1105 H, H-aliphatic).

Synthesis of S4: To a solution of $\mathbf{S 3}(400 \mathrm{mg}, 18 \mu \mathrm{mol})$ in $10 \mathrm{~mL}$ dry $\mathrm{CH}_{2} \mathrm{Cl}_{2}: \mathrm{MeOH}(1: 1)$ in a vial was added propargyl amine $(225 \mu \mathrm{L}, 180 \mathrm{mg}, 3 \mathrm{mmol})$ and the mixture stirred at room temperature. After $24 \mathrm{~h}$ more propargyl amine $(225 \mu \mathrm{L}, 180 \mathrm{mg}, 3 \mathrm{mmol})$ was added and the mixture stirred at room temperature for another $24 \mathrm{~h}$. The contents in the vial were transferred to a dialysis tube (Spectra/Por® Dialysis Membrane MWCO: 1000) and dialyzed against dichloromethane: $\mathrm{MeOH}(1: 1)(400 \mathrm{~mL})$ for 2 days with the solvent reservoir changed every 12 h. After dialysis, excess solvent was removed under vacuum to give $\mathbf{S 4}$ as a thin brown film. Yield 63\% yield; ${ }^{1} \mathrm{H}$ NMR (400 MHz, 1:1 CD 3 OD: DMSO-d6) $\delta$ 7.08-7.27 (m, 5 H, H-Ph) 5.365.21 (m, 196 H, H-olefinic) 3.78 (m, 202 H, -CH2-NH-), (m, 193, propargyl-H), 3.1-1.9 (m, 834 H, H-aliphatic).

Synthesis of S5 and S6: Azido Gal and Glc were prepared under the same conditions previously described. ${ }^{2,3}$ S5 was obtained a white solid. Yield 90\%; ' $\mathrm{H}$ NMR (400 MHz; CD $\left.3 \mathrm{OD}\right): \delta 4.45$ $(1 \mathrm{H}, \mathrm{d}, J=8.6 \mathrm{~Hz}, \mathrm{H}-1), 3.82(1 \mathrm{H}, \mathrm{d}, J=3.5 \mathrm{~Hz}, \mathrm{H}-4), 3.76(2 \mathrm{H}, \mathrm{m}, \mathrm{H}-6), 3.60$ (1H, t, J=8.8 Hz, H-5), 3.51 (2H, m, H-3, H-2).

S6 was obtained as a yellow syrup. Quantitative yield; ${ }^{1} \mathrm{H}$ NMR (400 MHz; CD $\left.{ }_{3} \mathrm{OD}\right): \delta 4.52(1 \mathrm{H}$, d, J=8.6 Hz, H-1), 3.91 (1H, dd, $J=12.0,2.0 \mathrm{~Hz}, \mathrm{H}-4), 3.71$ (1H, dd, $J=12.1,5.5$ Hz, H-5), 3.42$3.30(3 \mathrm{H}, \mathrm{m}, \mathrm{H}-6, \mathrm{H}-3), 3.17$ (1H, t, J=8.8 Hz, H-2)

Synthesis of Gal90 and Glc90: To a solution of S4 (25 mg, $1.6 \mu \mathrm{mol})$ in DMF (2 mL) in a vial was added a solution of either $\mathbf{S 5}$ or $\mathbf{S 6}(40 \mathrm{mg}, 0.2 \mathrm{mmol})$ in $\mathrm{MeOH}(2 \mathrm{~mL}), \mathrm{CuSO}_{4} \cdot 5 \mathrm{H}_{2} \mathrm{O}(8$ $\mathrm{mg}, 0.03 \mathrm{mmol})$ in $\mathrm{H}_{2} \mathrm{O}(0.5 \mathrm{~mL})$, sodium-ascorbate $(12 \mathrm{mg}, 0.01 \mathrm{mmol})$ in $\mathrm{H}_{2} \mathrm{O}(0.5 \mathrm{~mL})$ and 
the mixture stirred at $50{ }^{\circ} \mathrm{C} .1 \mathrm{~h}$ after completion of the reaction (approximately $3 \mathrm{~h}$ as judged by TLC with the click stain) $1 \mathrm{~g}$ of CupriSorb ${ }^{\circledR}$ was added and the mixture stirred overnight at 50 ${ }^{\circ} \mathrm{C}$. The CupriSorb ${ }^{\circledR}$ was filtered off and the filtrate transferred to a dialysis tube (Spectra/Por ${ }^{\circledR}$ Dialysis Membrane MWCO: 1000) and dialyzed against $\mathrm{H}_{2} \mathrm{O}(400 \mathrm{~mL})$ for 3 days with the solvent reservoir changed every $12 \mathrm{~h}$. After dialysis the solution was transferred to a scintillation vial and frozen in liquid nitrogen. The water was then removed by lyophilization to give either the Gal or Glc glycopolymer as a puffy white solid.

Gal90: Yield 49\%; 'H NMR (400 MHz, $\mathrm{D}_{2} \mathrm{O}$ ): $\delta 8.05$ (s (broad), $1 \mathrm{H}, \mathrm{H}$-Triazole) 5.57 (s; broad, 1 H, H1) 5.18 (s; broad, 2H, H-alkene) 3.98-3.52 (m, 8 H; H2, H3, H4, H5, H6, H6', -CH2), 3.39-0.39 (m, 7 H, H-aliphatic)

Glc90: Yield 63\%; 'H NMR (400 MHz, $\mathrm{D}_{2} \mathrm{O}$ ): $\delta 7.97$ (s (broad), 1 H, H-Triazole) 5.57 (s; broad, $1 \mathrm{H}, \mathrm{H1}) 5.16$ (s; broad, 2H, H-alkene) 4.26-3.59 (m, 8 H; H2, H3, H4, H5, H6, H6', - $\mathrm{CH}_{2}$ ), 3.10-0.523 (m, 7 H, H-aliphatic)

\section{References}

1. Okoth, R.; Basu, A., End-labeled amino terminated monotelechelic glycopolymers generated by ROMP and $\mathrm{Cu}(\mathrm{I})$-catalyzed azide-alkyne cycloaddition. Beilstein J. Org. Chem. 2013, $9,608-12$.

2. $\quad$ Tropper, F. D.; Andersson, F. O.; Braun, S.; Roy, R., Phase transfer catalysis as a general and stereoselective entry into glycosyl azides from glycosyl halides. Synthesis 1992, 618-620. 3. Rossi, L. L.; Basu, A., Glycosidase inhibition by 1-glycosyl-4-phenyl triazoles. Bioorg. Med. Chem. Lett. 2005, 15 (15), 3596-9. 